\title{
Combination Techniques and Decision Problems for Disunification
}

\author{
Franz Baader, Klaus Schulz
}

January 1993

Deutsches Forschungszentrum für Künstliche Intelligenz $\mathrm{GmbH}$

Postfach 2080

D-6750 Kaiserslautern, FRG

Tel.: (+49 631) 205-3211/13

Fax: (+49 631) 205-3210
Stuhlsatzenhausweg 3

D-6600 Saarbrücken 11, FRG

Tel.: (+49 681) 302-5252

Fax: (+49 681) 302-5341 


\section{Deutsches Forschungszentrum für Künstliche Intelligenz}

The German Research Center for Artificial Intelligence (Deutsches Forschungszentrum für Künstliche Intelligenz, DFKI) with sites in Kaiserslautern and Saarbrücken is a non-profit organization which was founded in 1988. The shareholder companies are Atlas Elektronik, Daimler-Benz, Fraunhofer Gesellschaft, GMD, IBM, Insiders, Mannesmann-Kienzle, SEMA Group, Siemens and SiemensNixdorf. Research projects conducted at the DFKI are funded by the German Ministry for Research and Technology, by the shareholder companies, or by other industrial contracts.

The DFKI conducts application-oriented basic research in the field of artificial intelligence and other related subfields of computer science. The overall goal is to construct systems with technical knowledge and common sense which - by using Al methods - implement a problem solution for a selected application area. Currently, there are the following research areas at the DFKI:

$\begin{array}{ll}\square & \text { Intelligent Engineering Systems } \\ \square & \text { Intelligent User Interfaces } \\ \square & \text { Computer Linguistics } \\ \square & \text { Programming Systems } \\ \square & \text { Deduction and Multiagent Systems } \\ \square & \text { Document Analysis and Office Automation. }\end{array}$

The DFKI strives at making its research results available to the scientific community. There exist many contacts to domestic and foreign research institutions, both in academy and industry. The DFKI hosts technology transfer workshops for shareholders and other interested groups in order to inform about the current state of research.

From its beginning, the DFKI has provided an attractive working environment for $\mathrm{Al}$ researchers from Germany and from all over the world. The goal is to have a staff of about 100 researchers at the end of the building-up phase.

Friedrich J. Wendl

Director 
Combination Techniques and Decision Problems for Disunification

Franz Baader, Klaus Schulz

DFKI-RR-93-05 
(C) Deutsches Forschungszentrum für Künstliche Intelligenz 1993

This work may not be copied or reproduced in whole or in part for any commercial purpose. Permission to copy in whole or in part without payment of fee is granted for nonprofit educational and research purposes provided that all such whole or partial copies include the following: a notice that such copying is by permission of Deutsches Forschungszentrum für Künstliche Intelligenz, Kaiserslautern, Federal Republic of Germany; an acknowledgement of the authors and individual contributors to the work; all applicable portions of this copyright notice. Copying, reproducing, or republishing for any other purpose shall require a licence with payment of fee to Deutsches Forschungszentrum für Künstliche Intelligenz. 


\title{
Combination Techniques and Decision Problems for Disunification
}

\author{
Franz Baader \\ DFKI \\ Stuhlsatzenhausweg 3 \\ 6600 Saarbrücken 11, Germany \\ e-mail: baader@dfki.uni-sb.de
}

\author{
Klaus U. Schulz \\ CIS \\ University Munich \\ 8000 München 40, Germany \\ e-mail: schulz@sun1.cis.uni-muenchen.de
}

\begin{abstract}
Previous work on combination techniques considered the question of how to combine unification algorithms for disjoint equational theories $E_{1}, \ldots, E_{n}$ in order to obtain a unification algorithm for the union $E_{1} \cup \ldots \cup E_{n}$ of the theories. Here we want to show that variants of this method may be used to decide solvability and ground solvability of disunification problems in $E_{1} \cup \ldots \cup E_{n}$. Our first result says that solvability of disunification problems in the free algebra of the combined theory $E_{1} \cup \ldots \cup E_{n}$ is decidable if solvability of disunification problems with linear constant restrictions in the free algebras of the theories $E_{i}(i=1, \ldots, n)$ is decidable. In order to decide ground solvability (i.e., solvability in the initial algebra) of disunification problems in $E_{1} \cup \ldots \cup E_{n}$ we have to consider a new kind of subproblem for the particular theories $E_{i}$, namely solvability (in the free algebra) of disunification problems with linear constant restriction under the additional constraint that values of variables are not $E_{i}$-equivalent to variables. The correspondence between ground solvability and this new kind of solvability holds, (1) if one theory $E_{i}$ is the free theory with at least one function symbol and one constant, or (2) if the initial algebras of all theories $E_{i}$ are infinite. Our results can be used to show that the existential fragment of the theory of the (ground) term algebra modulo associativity of a finite number of function symbols is decidable; the same result follows for function symbols which are associative and commutative, or associative, commutative and idempotent.
\end{abstract}




\section{Contents}

1 Introduction $\quad 3$

2 Formal Preliminaries $\quad 4$

3 Solvability of Disunification Problems $\quad 6$

4 Ground Solvability $\quad 13$

5 Applications of the Method 20

5.1 Ground Solvability for A . . . . . . . . . . . . 24

5.2 Ground Solvability for $A C$. . . . . . . . . . . . 25

5.3 Ground Solvability for ACI . . . . . . . . . . . . . 26

$\begin{array}{llr}6 & \text { Conclusion } & 27\end{array}$ 


\section{Introduction}

In recent years the rôle Robinson unification - and later unification modulo equational theories-played in theorem proving, term rewriting, and logic programming has more and more been taken on by constraint solving (see e.g., [Bür90, KK89, JL87, Col90]). One advantage of constraint approaches is that it is no longer necessary to compute (a complete set of) solutions; deciding satisfiability of the constraints is usually sufficient. Thus one can, for example, work modulo non-finitary equational theories such as associativity. Another motivation for preferring a constraint approach is that in this setting the expressive power of a formalism can rather naturally be enhanced by considering more general constraints than the equality constraints of unification problems. One of the earliest of these generalizations was Colmerauer's use of equations and negated equations in PROLOG II [Col84]. In the present paper we shall consider solvability of this kind of equational problems (subsequently called disunification problems) modulo equational theories.

As for unification, the terms in the disunification problems occurring in applications are usually not just built over the signature of the equational theory, but they contain additional free function symbols. More generally, one often wants to solve disunification problems containing function symbols whose properties are defined by different equational theories. For the case of unification, this fact has triggered extensive research on the combination of unification procedures for disjoint equational theories (see, e.g., the introduction of [BS91a] for a brief overview), but until now these approaches have not been generalized to the disunification case. One reason is that until recently the combination methods were restricted to equational theories which are finitary unifying, i.e., they combined algorithms computing finite complete sets of unifiers. In this setting, solvability of disunification problems can be reduced to the unification and the word problem for the equational theory. In fact, to decide solvability of a disunification problem, one simply computes a finite complete set of unifiers for the equations of the problem, and then checks whether one of these unifiers is a solution of the whole disunification problem. This means that for finitary theories it is sufficient to have combination methods for unification. However, if one only has a procedure that decides satisfiability of unification problems, such a reduction of disunification to unification does not seem to be possible. In addition, even if a theory is finitary, the computation of a complete set of unifiers can be of higher complexity than deciding solvability (associativity and commutativity is an example for this phenomenon).

In [BS91a] we have shown how to combine decision procedures for unification, and in the present paper we shall investigate how this method can be generalized to treat solvability of disunification problems. For unification, "solvability" means having a solution in the free algebra (in countably many generators), or equivalently, having a solution in the initial algebra. For disunification, solvability in 
the initial algebra (called ground solvability in the following) implies solvability in the free algebra (simply called solvability below), but not vice versa. Both types of solvability are considered in the literature (see [Com91, Bür88]), but ground solvability seems to be more interesting for most applications.

For solvability, the adaptation of the combination method to disunification problems is relatively straightforward. The main tool of the method is a decomposition algorithm which transforms every disunification problem $\Gamma$ in the combination of arbitrary disjoint equational theories $E_{1}, \ldots, E_{n}$ into a finite number of tuples $\left(\Gamma_{1}, \ldots, \Gamma_{n}\right)$, where each $\Gamma_{i}$ is an $E_{i}$-disunification problem with linear constant restriction. ${ }^{1}$ As for unification, $\Gamma$ is solvable in the combined theory iff for one of these tuples all its components are solvable in the single theories. However, the proof of soundness of the method - which is almost trivial for the case of unification problems - becomes a lot more involved.

For the ground case, it surprisingly turned out that ground solvability of $\Gamma$ in the combined theory is not reduced by our method to ground solvability of the components of one of the tuples in the single theories. On the contrary, one has to consider a slightly restricted form of solvability (in the free algebra in countably many generators) for the $E_{i}$-disunification problem with linear constant restriction $\Gamma_{i}$. It should also be noted that for ground solvability to be handled by our method the equational theories have to satisfy an additional condition. This condition holds, however, in various situations which are interesting for applications (see Section 5).

The paper has the following structure. The next section starts with some technical preliminaries. In Section 3 we introduce the decomposition algorithm, show its correctness for the case of solvability, and state some consequences. Section 4 is concerned with ground solvability, and in Section 5 the results are applied to combine disunification algorithms for the free theory and the theories $A$ (associativity), AC (associativity and commutativity), and ACI (associativity, commutativity, and idempotence).

\section{Formal Preliminaries}

For an equational theory $E$, let $\operatorname{sig}(E)$ denote its signature, i.e., the function symbols occurring in the identities of $E$. We assume that this signature is finite. For disunification it is even more important than for unification to know the signature over which the terms in the formulation of the problem and in the solutions of the problem may be built. For this reason, we shall explicitly talk about $(E, \Sigma)$-disunification problems, where $\Sigma$ is a finite superset of $\operatorname{sig}(E)$. Such

\footnotetext{
${ }^{1}$ This is the obvious adaptation of the notion "unification problem with linear constant restriction," as introduced in [BS91a]; see Section 2 for a definition.
} 
a problem is a finite set of equations and disequations

$$
\Gamma=\left\{s_{1} \doteq t_{1}, \ldots, s_{n} \doteq t_{n}\right\} \cup\left\{s_{n+1} \neq t_{n+1}, \ldots, s_{n+m} \neq t_{n+m}\right\},
$$

where $s_{1}, \ldots, t_{n+m}$ are $\Sigma$-terms. A solution of the $(E, \Sigma)$-disunification problem $\Gamma$ is a $\Sigma$-substitution $\sigma$ such that $s_{i} \sigma={ }_{E} t_{i} \sigma(i=1, \ldots, n)$ and $s_{n+j} \sigma \neq_{E} t_{n+j} \sigma$ $(j=1, \ldots, m)$. A ground solution is a solution that maps all variables occurring in $\Gamma$ to variable-free $\Sigma$-terms. $\Gamma$ is called (ground) solvable iff it has a (ground) solution.

It should be noted that the notion of a disunification problem does not always refer to the same kind of problem in the literature. Our definition coincides with the one of Bürckert [Bür88], who considers existentially quantified equational formulae, but other authors (e.g., Comon [Com91]) allow for arbitrary quantification.

As in the case of unification, one has to distinguish several types of disunification problems. The $(E, \Sigma)$-disunification problem is called elementary, if $\Sigma=\operatorname{sig}(E)$; it is a disunification problem with constants, if $\Sigma \backslash \operatorname{sig}(E)$ is a finite set of constants; and it is a general disunification problem, if no such restrictions hold.

Solvability of an $(E, \Sigma)$-disunification problem obviously means that the equations and disequations can be solved in the $E$-free $\Sigma$-algebra $T(\Sigma, Y) /={ }_{E}$ over the countable set of variables $Y$, whereas ground solvability means that they can be solved in the initial algebra $T(\Sigma, \emptyset) /=_{E}$. If one has no disequations (i.e., one has a unification problem), then both notions coincide, but this is not the case if disequations are present. For example, let $E$ be the empty theory, and assume that $\Sigma$ consists of the constant symbol $a$. The $(E, \Sigma)$-disunification problem $\{x \neq a\}$ is solvable, but not ground solvable.

The combination problem for disunification can now formally be defined as follows. Let $E_{1}, E_{2}$ be two equational theories built over the disjoint signatures $\Sigma_{1}:=\operatorname{sig}\left(E_{1}\right)$ and $\Sigma_{2}:=\operatorname{sig}\left(E_{2}\right),{ }^{2}$ and let $E=E_{1} \cup E_{2}$ denote their union. We are interested in solving elementary disunification problems for $E$, i.e., $\left(E, \Sigma_{1} \cup \Sigma_{2}\right)$ disunification problems. The terms in such problems are built from variables and symbols of $\Sigma_{1} \cup \Sigma_{2}$. The elements of $\Sigma_{1}$ will be called 1-symbols and the elements of $\Sigma_{2} 2$-symbols. A term $t$ is called $i$-term iff it is of the form $t=f\left(t_{1}, \ldots, t_{n}\right)$ for an $i$-symbol $f(i=1,2)$. A subterm $s$ of a 1 -term $t$ is called alien subterm of $t$ iff it is a 2-term such that every proper superterm of $s$ in $t$ is a 1-term. Alien subterms of 2 -terms are defined analogously. An $i$-term $s$ is pure iff it contains only $i$-symbols and variables. A (dis)equation $s \doteq t(s \neq t)$ is pure iff there exists an $i, 1 \leq i \leq 2$, such that $s$ and $t$ are pure $i$-terms or variables; this (dis)equation is then called an $i$-(dis)equation. Please note that according to this definition equations of the form $x \doteq y$ where $x$ and $y$ are variables are both 1- and 2-equations, and similarly

\footnotetext{
${ }^{2}$ We shall restrict the technical presentation to the combination of two theories. The combination of more than two theories can be treated analogously.
} 
for disequations. In the following, the symbols $x, y, z$, with or without indices, will always stand for variables.

Solvability of elementary disunification problems in $E$ will be reduced to solvability of disunification problems with constants in the single theories $E_{1}, E_{2}$. But as in the unification case, the solutions of these problems with constants have to satisfy additional restrictions. These restrictions are formalized in the notion of a disunification problem with linear constant restriction. For an equational theory $F$ with signature $\Omega$, such a problem consists of two parts:

1. An $(F, \Omega \cup C)$-disunification problem $\Gamma$, where $C$ is a finite set of constant symbols not occurring in $\Omega$, and

2. a linear ordering $<$ on $C \cup X$, where $X$ is a finite superset of the set of variables occurring in $\Gamma$.

For a given problem of this kind, the sets $V_{c}$ of variables which must not use $c$ are defined as $V_{c}:=\{x \in X ; x<c\}$, for every $c \in C$. A solution of the problem is a substitution $\sigma$ which assigns terms $x \sigma$ built with variables, symbols from $\Omega$, and constants in $C$ to the variables $x \in X$, solves all equations and disequations of $\Gamma$ modulo $F$, and has the additional property that $c$ does not occur in $x \sigma$ for all $c \in C$ and $x \in V_{c}$. A solution $\sigma$ is called restrictive if for all variables $x \in X$ the value $x \sigma$ is not $F$-equivalent to a variable. Restrictive solutions will become important if one is interested in ground solvability in the combined theory $E$.

Disunification problems with linear constant restriction will be denoted in the form $(\Gamma, X, C,<)$, or just as $\Gamma$, if no misleading ambiguities are possible.

\section{Solvability of Disunification Problems}

Our first main result says that solvability of disunification problems in the combination of disjoint equational theories can be reduced to solvability of disunification problems with linear constant restriction in the single theories.

Theorem 3.1 Let $E_{1}, \ldots, E_{n}$ be equational theories over disjoint signatures such that solvability of disunification problems with linear constant restriction is decidable for $E_{1}, \ldots, E_{n}$. Then solvability of elementary disunification problems is decidable for the combined theory $E_{1} \cup \ldots \cup E_{n}$.

This result is analogous to the one for unification given in [BS91a], and it depends on a decomposition algorithm which is very similar to the algorithm presented in that paper. However, the proof of soundness of the method is more complex. As mentioned above, we shall restrict the presentation to the combination of two theories. 


\section{The Decomposition Algorithm}

The input for this algorithm is an elementary $E$-disunification problem, i.e., a system

$$
\Gamma_{0}=\left\{s_{1} \doteq t_{1}, \ldots, s_{n} \doteq t_{n}, s_{n+1} \not t_{n+1}, \ldots, s_{n+m} \not t_{n+m}\right\},
$$

where the terms $s_{1}, \ldots, t_{n+m}$ are built from variables and the function symbols occurring in $\Sigma_{1} \cup \Sigma_{2}$, the signature of $E=E_{1} \cup E_{2}$. The first two steps of the algorithm are deterministic, i.e., they transform the given system into one new system.

\section{Step 1: variable abstraction.}

Alien subterms are successively replaced by new variables until all terms occurring in the system are pure. To be more precise, assume that $s \doteq t$ or $t \doteq s(s \neq t$ or $t \neq s$ ) is an equation (disequation) in the current system, and that $s$ contains the alien subterm $s_{1}$. Let $x$ be a variable not occurring in the current system, and let $s^{\prime}$ be the term obtained from $s$ by replacing $s_{1}$ by $x$. Then the original equation (disequation) is replaced by the two equations $s^{\prime} \doteq t$ and $x \doteq s_{1}$ (by the disequation $s^{\prime} \neq t$ and the equation $\left.x \doteq s_{1}\right)$. This process has to be iterated until all terms occurring in the system are pure.

\section{Step 2: split non-variable disequations and non-pure equations.}

Each disequation of the form $s \neq t$ (where $s$ or $t$ is not a variable) is replaced by two equations $x \doteq s, y \doteq t$ and a disequation $x \neq y$ where the $x, y$ are always new variables. Each non-pure equation of the form $s \doteq t$ is replaced by two equations $x \doteq s, x \doteq t$ where the $x$ are always new variables.

It is quite obvious that these two steps do not change solvability of the system. The result is a system which consists of pure equations and of disequations between variables. The third and the fourth step are nondeterministic, i.e., a given system is transformed into finitely many new systems. Here the idea is that the original system is solvable iff at least one of the new systems is solvable.

\section{Step 3: variable identification.}

Consider all partitions of the set of all variables occurring in the system such that distinct variables $x, y$ are in distinct classes of the partition if the system contains the disequation $x \neq y$. Each of these partitions yields one of the new systems as follows. The variables in each class of the partition are "identified" with each other by choosing an element of the class as representative, and replacing in the system all occurrences of variables of the class by this representative. In addition, we add a disequation $x \neq y$ for every pair $x, y$ of distinct representatives to the system if this disequation is not already present. 


\section{Step 4: choose ordering and theory indices.}

This step does not modify a given system, it just adds some information which will be important in the next step. For a given system, consider all possible strict linear orderings $<$ on the variables of the system, and all mappings ind from the set of variables into the set of theory indices $\{1,2\}$. Each pair $(<$, ind $)$ yields one of the nèw systems obtained from the given one.

For a system obtained by Step 4 , let $X_{5, i}$ denote the set of variables of index $i(i=1,2)$. The last step is again deterministic. It splits each of the systems already obtained into a pair of pure systems.

\section{Step 5: split systems.}

A given system $\Gamma$ is split into two systems $\Gamma=\Gamma_{1} \cup \Gamma_{2}$ such that $\Gamma_{1}$ contains only 1-(dis)equations and $\Gamma_{2}$ only 2 -(dis)equations. As an additional restriction, the system $\Gamma_{i}(i=1,2)$ must contain all disequations $x \neq y$ where $x$ or $y$ has index $i$. This means that disequations between variables of distinct indices are put into both subsystems. The subsystems can now be considered as disunification problems with linear constant restriction $\left(\Gamma_{1}, X_{5,1}, X_{5,2},<\right)$ and $\left(\Gamma_{2}, X_{5,2}, X_{5,1},<\right)$ which have to be solved modulo $E_{1}$ and $E_{2}$, respectively. This means that in the system $\Gamma_{i}$ the variables with index $i$ are still treated as variables, but the variables with alien index $j \neq i$ are treated as free constants.

The output of the algorithm is thus a finite set of pairs $\left(\Gamma_{1}, \Gamma_{2}\right)$ where the first component $\Gamma_{1}$ is an $\left(E_{1}, \Sigma_{1} \cup X_{5,2}\right)$-disunification problem with linear constant restriction, and the second component $\Gamma_{2}$ is an $\left(E_{2}, \Sigma_{2} \cup X_{5,1}\right)$-disunification problem with linear constant restriction.

There are three points where this decomposition algorithm is not a totally straightforward adaptation of the one for unification problems. First, we split all non-variable disequations and not only the non-pure ones. This greatly facilitates the proof of correctness of the method, but is not mandatory. Second, we add disequations between all variables which have not been identified with each other in Step 3, and third, disequations involving variables of index $i$ are required to be in $\Gamma_{i}$ in Step 5. The latter two points are necessary for the following proposition to hold.

Proposition 3.2 The input system $\Gamma_{0}$ is solvable if and only if there exists a pair $\left(\Gamma_{1}, \Gamma_{2}\right)$ in the output set such that $\Gamma_{1}$ and $\Gamma_{2}$ are solvable.

The proposition shows that the decomposition algorithm can be used to reduce solvability of elementary disunification problems for $E_{1} \cup E_{2}$ to solvability of disunification problems with linear constant restriction for $E_{1}, E_{2}$. Thus Theorem 3.1 is an immediate consequence of Proposition 3.2. Before we give a proof of the proposition, let us mention some additional consequences. 
Corollary 3.3 (1) Let $E$ be an equational theory such that solvability of disunification problems with linear constant restriction is decidable. Then solvability of general E-disunification problems is decidable.

(2) The result of Theorem 3.1 can be lifted to general disunification problems, i.e., the assumptions of Theorem 3.1 are sufficient to get decidability of general disunification problems in the combined theory.

(3) If, for $E_{1}$ and $E_{2}$, solvability of disunification problems with linear constant restriction can be decided by an NP-algorithm, then solvability of disunification problems in the combined theory is also NP-decidable.

Proof. The proof is very similar to the one given in [BS91a, BS91b] for the analogous results for unification problems.

(1) Let $\Gamma$ be a general $(E, \Sigma)$-disunification problem, and let $\Omega:=\Sigma \backslash \operatorname{sig}(E)$. The system $\Gamma$ may be considered as a disunification problem in the union of the theory $E$ with the free theory

$$
F_{\Omega}:=\left\{f\left(x_{1}, \ldots, x_{n}\right)=f\left(x_{1}, \ldots, x_{n}\right) \mid f \in \Omega\right\} .
$$

Obviously, the relation $={ }_{F_{\Omega}}$ is just the syntactic equality of terms.

By Theorem 3.1 it remains to be shown that solvability of $F_{\Omega}$-disunification problems with linear constant restriction is decidable. But this is very easy. For an $F_{\Omega}$-disunification problem with linear constant restriction, $\Gamma_{2}$, one first computes a most general unifier $\sigma$ of the equations in $\Gamma_{2}$. The whole system $\Gamma_{2}$ has a solution iff $\sigma$ solves $\Gamma_{2}$, i.e., if $\sigma$ respects the linear constant restriction, and does not identify the two sides of a disequation of $\Gamma_{2}$.

In fact, any solution of $\Gamma_{2}$ is a solution of the equations in $\Gamma_{2}$, and thus an instance of $\sigma$. If $\sigma$ does not satisfy the constant restriction (i.e., $c$ occurs in $x \sigma$ for $x<c$ ) then no instance of $\sigma$ will satisfy the constant restriction (for any substitution $\lambda$ we have that $c$ occurs in $x \sigma \lambda$ ). The same is true if $\sigma$ does not solve a disequation $s \neq t$ of $\Gamma_{2}$ (since $s \sigma=t \sigma$ implies $\left.s \sigma \lambda=t \sigma \lambda\right)$.

(2) Let $E=E_{1} \cup \cdots \cup E_{n}$, and $\Sigma=\operatorname{sig}\left(E_{1}\right) \cup \cdots \cup \operatorname{sig}\left(E_{n}\right) \cup \Omega$. In order to get decidability of $(E, \Sigma)$-disunification problems one just applies Theorem 3.1 to the combination of $E_{1}, \ldots, E_{n}$, and $F_{\Omega}$.

(3) It is easy to see that the decomposition algorithm is an NP-algorithm (see [BS91b] for a detailed analysis for the case of unification problems). The resulting systems $\Gamma_{5,1}, \Gamma_{5,2}$ are of a size that is polynomial in the size of the original system. If deciding whether these systems are solvable can also be done by an NP-algorithm, then the overall decision method is an NP-algorithm.

In order to prove Proposition 3.2, some technical background is needed. Without loss of generality, we make the general assumption that all equational theories which are considered are consistent. Now let $E_{1}, E_{2}$ be equational theories over 
disjoint signatures $\Sigma_{1}, \Sigma_{2}$. Let $T\left(\Sigma_{1} \cup \Sigma_{2}, Y\right)$ be the set of all terms built over the signatures $\Sigma_{1}, \Sigma_{2}$ with variables in $Y$. Applying unfailing completion (see e.g., [DJ89]) to the combined theory $E=E_{1} \cup E_{2}$, but always treating the elements $y \in Y$ as constants, we obtain a possibly infinite ordered-rewriting system $R$ which is confluent and terminating on $T\left(\Sigma_{1} \cup \Sigma_{2}, Y\right)$. Thus we eventually obtain, applying $R$, a unique irreducible normal form $t_{\downarrow R}$ for every term $t \in T\left(\Sigma_{1} \cup \Sigma_{2}, Y\right)$. We denote the set of $R$-irreducible elements of $T\left(\Sigma_{1} \cup \Sigma_{2}, Y\right)$ by $T_{\mid R}$.

We want to establish a relationship between impure terms and corresponding pure terms where alien subterms have been replaced by new variables. For this purpose, we consider a bijection $\pi: T_{\downarrow R} \longrightarrow Z$ where $Z$ is a set of variables of appropriate cardinality. This bijection induces mappings $\pi_{i}$ of terms in $T\left(\Sigma_{1} \cup\right.$ $\left.\Sigma_{2}, Y\right)$ to terms in $T\left(\Sigma_{i}, Z\right)$ as follows. For variables $y \in Y, y^{\pi_{i}}:=\pi(y)$ (note that variables are always $R$-irreducible.) If $t=f\left(t_{1}, \ldots, t_{n}\right)$ for an $i$-symbol $f$, then $t^{\pi_{i}}:=f\left(t_{1}^{\pi_{i}}, \ldots, t_{n}^{\pi_{i}}\right)$. Finally, if $t$ is a $j$-term, $j \neq i$, then $t^{\pi_{i}}:=\pi\left(t_{\mid R}\right)$. The mapping $\pi_{i}$ may be regarded as a projection which maps a possibly mixed term to a pure $i$-term or a variable.

- A substitution $\sigma$ is called $R$-normalized on a finite set of variables $X$ iff $x \sigma \in$ $T_{\downarrow R}$ for all variables $x \in X$. The next lemma was proved-under almost the same assumptions - in [BS91a]: there we additionally assumed that $Y$ and $Z$ are disjoint; but the proof of the lemma does not depend on this property.

Lemma 3.4 Let $s, t$ be pure i-terms or variables, and let $\sigma$ be a substitution which is R-normalized on the variables occurring in $s, t$. Then

$$
s \sigma=E_{E} t \sigma \quad \text { iff }(s \sigma)^{\pi_{i}}=E_{E_{i}}(t \sigma)^{\pi_{i}} .
$$

\section{Proof of Proposition 3.2}

Here and in the remainder of this paper, $\Gamma_{0}$ always denotes an input system of the combination algorithm, $\Gamma_{j}$ denotes (one of) the system(s) obtained from $\Gamma_{0}$ after Step $j$ of the algorithm $(j=1,2,3,4)$. The two subsystems obtained after Step 5 are denoted by $\Gamma_{5, i}(i=1,2)$. $X_{j}$ denotes the set of variables occurring in $\Gamma_{j}(j=0, \ldots, 4)$ and $X_{5, i}$ denotes the variables $x \in X_{4}$ with index $i(i=1,2)$. Thus $X_{4}=X_{5,1} \cup X_{5,2}$, and this is a disjoint union.

The proof of completeness (i.e., of the "only if" part of the proposition) is very similar to the one for the unification case (see [BS91a], proof of Proposition 3.2). Let $\sigma$ be a solution of $\Gamma_{0}$. Without loss of generality we may assume that $\sigma$ also solves the system $\Gamma_{2}$ obtained after Step 2 of the decomposition algorithm, and that $\sigma$ is $R$-normalized on $X_{2}$.

The solution $\sigma$ can be used to define the correct alternatives in the nondeterministic steps of the algorithm:

- The partition of $X_{2}$ in the third step is defined as follows. Two variables 
$x$ and $y$ are in the same class iff $x \sigma=y \sigma$. (Obviously, this means that $\sigma$ is also a solution of the system $\Gamma_{3}$ obtained after the variable identification step corresponding to this partition.)

- In the fourth step, the variable $x$ gets index $i$ if $x \sigma$ is an $i$-term. If $x \sigma$ is itself a variable, $x$ gets index 1 . (This is arbitrary, we could have taken index 2 as well.)

- To get an appropriate linear ordering in the fourth step, we consider the strict partial ordering defined by $x<y$ iff $x \sigma$ is a strict subterm of $y \sigma$. Now we take an arbitrary extension of this partial ordering to a linear ordering on $X_{3}=X_{4}$.

These choices determine systems $\Gamma_{3}, \Gamma_{4}$ and a pair $\left(\Gamma_{5,1}, \Gamma_{5,2}\right)$ in the output set of the combination algorithm. It remains to be shown that, $\Gamma_{5,1}, \Gamma_{5,2}$ are solvable. In order to define solutions $\sigma_{i}$ of these systems, we consider a bijection $\pi$ from $T_{\downarrow R}$ onto a set of variables $Z$ containing $X_{4}$ such that $\pi(x \sigma)=x$ for all $x \in X_{4}$. The substitution $\sigma_{i}$ is defined on the variables $x \in X_{4}$ by

$$
x \sigma_{i}:=(x \sigma)^{\pi_{i}},
$$

where $\pi_{i}$ is the $i$-projection induced by $\pi$. In [BS91a] (proof of Proposition 3.2) it is shown that, for $i=1,2$, the substitution $\sigma_{i}$ treats variables $x \in X_{5, j}(j \neq i)$ as constants, respects the linear constant restriction, and solves all equations of $\Gamma_{5, i}$. What remains to be shown here is that the disequations in $\Gamma_{5, i}$ are satisfied as well. But these are just disequations between distinct variables in the system, i.e., of the form $x \neq y$ for distinct variables $x, y \cdot \in X_{4}$. By the choice made in the variable identification step we know that $x \sigma \neq_{E} y \sigma$. But then

$$
x \sigma_{i}=(x \sigma)^{\pi_{i}} \neq E_{i}(y \sigma)^{\pi_{i}}=y \sigma_{i},
$$

by Lemma 3.4. This shows that $\sigma_{i}$ solves the disequations of $\Gamma_{5, i}$ as well.

To show soundness (i.e., the "if" part of the proposition) we have to demonstrate that $\Gamma_{0}$ is solvable if there exists a pair $\left(\Gamma_{5,1}, \Gamma_{5,2}\right)$ in the output set such that $\Gamma_{5,1}$ and $\Gamma_{5,2}$ are solvable. In the unification case, this part was almost trivial, but it is a lot more complex here.

Let $\sigma_{1}$ be a solution of $\Gamma_{5,1}$ and $\sigma_{2}$ a solution of $\Gamma_{5,2}$. We may assume that $\sigma_{i}: X_{5, i} \rightarrow T\left(\Sigma_{i} \cup X_{5, j}, Y_{i}\right)(i, j \in\{1,2\}, i \neq j)$, where $Y_{1}, Y_{2}$ are two disjoint, infinite sets of variables such that $X_{4}$ and their union $Y:=Y_{1} \cup Y_{2}$ are disjoint. Let $R$ be a possibly infinite ordered-rewriting system $R$ which is confluent and terminating on $T\left(\Sigma_{1} \cup \Sigma_{2}, Y\right)$ (obtained by unfailing completion, as described above).

Using $R$ and the $\sigma_{i}$ we shall now define a substitution $\sigma$ on $X_{4}$ which solves $\Gamma_{4}$. It is then trivial to extend $\sigma$ to a solution of $\Gamma_{0}$. In order to define $\sigma$ we 
proceed along the linear order $<$ which was chosen in Step 4 of the algorithm. Assume that $z \sigma \in T_{\downarrow R}$ has been defined for all $z<x$. Without loss of generality we assume that $x$ has index 1 . Since $\sigma_{1}$ satisfies the linear constant restriction associated with $<$, we know that all $z \in X_{5,2}$ occurring in $x \sigma_{1}$ are smaller than $x$ with respeçt to $<$. For this reason, $x \sigma:=\left(x \sigma_{1} \sigma\right)_{\downarrow R}$ is well-defined.

In the corresponding definition for the unification case, the term $x \sigma_{1} \sigma$ was not $R$-reduced. This means that the substitution we defined there is not identical to the one defined here, but obviously the two substitution are $E$-equivalent. For this reason the proof given in [BS91a] to show that $\sigma$ solves the original unification problem can be taken without change to show that $\sigma$ solves the equations in $\Gamma_{4}$.

The following two claims, which will be proved by induction on the linear order $<$, establish that $\sigma$ solves the disequations as well.

(C1) for all $x_{1}, x_{2} \in X_{4}$ with $x_{1} \neq x_{2}$ we have $x_{1} \sigma \neq_{E} x_{2} \sigma$,

(C2) for each $x_{1} \in X_{4}$ : if ind $\left(x_{1}\right)=i$, then $x_{1} \sigma \in T_{\downarrow R}$ is an $i$-term or an element of $Y_{i}$.

Without loss of generality, let us consider an element $x$ of index 1 . The induction hypothesis that we may use is that Conditions (C1) and (C2) are valid for all $x_{1}, x_{2}<x$. We shall now show that the same is true for all $x_{1}, x_{2} \leq x$. Let $X_{5,2}^{<x}=\left\{x_{1} \in X_{5,2} ; x_{1}<x\right\}$. We consider a bijection

$$
\pi_{x}: T_{\downarrow R} \longrightarrow Y \cup X_{5,2}^{<x} \cup Z
$$

where $Z$ is a set of new variables. This bijection has to satisfy the following conditions:

1. $\pi_{x}(t) \in Y_{2} \cup X_{5,2}^{<x} \cup Z$ for every 2 -term $t \in T_{\downarrow R}$,

2. $\pi_{x}(y) \in Y_{2} \cup X_{5,2}^{<x} \cup Z$ for every $y \in Y_{2}$,

3. $\pi_{x}(y)=y$ for every $y \in Y_{1}$,

4. $\pi_{x}\left(x_{2} \sigma\right)=x_{2}$ for every $x_{2} \in X_{5,2}^{<x}$.

It is easy to see that the induction hypothesis guarantees the existence of such a bijection, provided that $Z$ is chosen of appropriate cardinality.

First, let us show that $x \sigma$ is a 1-term in $T_{\downarrow R}$ or an element of $Y_{1}$, thus verifying Condition (C2). Obviously $x \sigma \in T_{\downarrow R}$. Let $\sigma_{x}$ denote the restriction of $\sigma$ to the variables $z \leq x$ of $X_{4}$. By induction hypothesis, $\sigma_{x}$ is $R$-normalized and we have

$$
x \sigma_{1} \sigma_{x}=x \sigma_{1} \sigma={ }_{E}\left(x \sigma_{1} \sigma\right)_{\downarrow R}=x \sigma=x \sigma_{x} .
$$


By Lemma 3.4 we get

$$
\left(x \sigma_{1} \sigma_{x}\right)^{\pi_{1}}=E_{1}\left(x \sigma_{x}\right)^{\pi_{1}},
$$

where $\pi_{1}$ is the 1 -projection determined by $\pi_{x}$. Now let us show that $\left(x \sigma_{1} \sigma_{x}\right)^{\pi_{1}}=$ $x \sigma_{1}$. If $x \sigma_{1} \in Y_{1}$, this equality obviously holds since $\sigma_{x}$ and $\pi_{1}$ do not move these variables, by Condition 3 on $\pi_{x}$. If $x \sigma_{1}$ is a 1-term, then the "constants" $x_{2} \in X_{5,2}$ occurring in this term are in $X_{5,2}^{<x}$. Now $\sigma_{x}$ substitutes for these constants $R$ irreducible 2-terms or elements of $Y_{2}$, by induction hypothesis (C2). In both cases, $\pi_{1}$ will reintroduce the old constants again, by Condition 4 on $\pi_{x}$. The variables $y \in Y_{1}$ occurring in $x \sigma_{1}$ are not touched, neither by $\sigma_{x}$ nor by $\pi_{1}$. Therefore the equality holds again. By our assumption on $\sigma_{1}$ it remains the case where $x \sigma_{1}=x_{2} \in X_{5,2}$. But this case cannot occur since $\sigma_{1}$ solves the disequation $x \neq x_{2} \in \Gamma_{5,1}$, and since $x_{2} \sigma_{1}=x_{2}$. Combining what we have found so far we get

$$
x \sigma_{1}=E_{1}\left(x \sigma_{x}\right)^{\pi_{1}} .
$$

Now suppose that $x \sigma_{x}$ is a 2-term or an element of $Y_{2}$. Then $\left(x \sigma_{x}\right)^{\pi_{1}}=y$ would be an element of $Y_{2} \cup X_{5,2}^{<x} \cup Z$, by Conditions 1 and 2 on $\pi_{x}$, and we have $x \sigma_{1}=E_{E_{1}} y$. But $x \sigma_{1}$ contains only variables from $Y_{1} \cup X_{5,2}^{<x}$. Since $E_{1}$ is consistent, $y$ cannot be an element of $Z \cup Y_{2}$. For $y \in X_{5,2}^{<x}$ we get $x \sigma_{1}=_{E_{1}} y=y \sigma_{1}$. But this is again impossible since $\sigma_{1}$ solves the system $\Gamma_{5,1}$, which contains the disequation $x \neq y$.

By excluding all other cases we have shown that $x \sigma=x \sigma_{x}$ is a 1-term or a variable in $Y_{1}$. Thus $(\mathrm{C} 2)$ is verified.

Now let us consider Condition (C1). Let $z<x$ and assume that $z \sigma={ }_{E}$ $x \sigma$. Since both terms are $R$-irreducible we have even $z \sigma=x \sigma$. The induction hypothesis and Condition (C2) for $x$ show that $z$ cannot have index 2 since $Y_{1}$ and $Y_{2}$ are disjoint. Thus $x$ and $z$ both have index 1 , and we get.

$$
z \sigma_{1} \sigma={ }_{E} z \sigma=x \sigma={ }_{E} z \sigma_{1} \sigma .
$$

By definition of $\pi_{x}$ we have $\left(z \sigma_{1} \sigma\right)^{\pi_{1}}=z \sigma_{1}$ and $\left(x \sigma_{1} \sigma\right)^{\pi_{1}}=x \sigma_{1}$, as we have seen earlier for $x$. With Lemma 3.4 we obtain $z \sigma_{1}=E_{1} x \sigma_{1}$, which is a contradiction since $x \neq z \in \Gamma_{5,1}$. This concludes the proof of the two claims.

Since all disequations in $\Gamma_{4}$ are disequations between variables, (C1) implies that $\sigma$ solves these disequations.

\section{Ground Solvability}

The preceding section shows that, analogously to the unification case, solvability of disunification problems in the combined theory can be reduced by decomposition to solvability of disunification problems with linear constant restriction in the single theories. An obvious conjecture could be that the same holds for ground 
solvability, i.e., that ground solvability of a disunification problem $\Gamma_{0}$ may be decided by decomposing $\Gamma_{0}$ into a finite set of pairs $\left(\Gamma_{5,1}, \Gamma_{5,2}\right)$ of $E_{i}$-disunification problems with linear constant restriction as described above, and then asking for ground solvability of the subproblems. However, this method is only sound, but not complete.

Proposition 4.1 Let $\Gamma_{0}$ be an input problem of the decomposition algorithm. Suppose that there exists an output pair $\left(\Gamma_{5,1}, \Gamma_{5,2}\right)$ such that each $\Gamma_{5, i}(i=1,2)$ has a ground solution. Then $\Gamma_{0}$ has a ground solution.

Proof. Assume that the substitution $\sigma$ is constructed from ground solutions of $\Gamma_{5,1}$ and $\Gamma_{5,2}$ as described in the proof of the "if" part of Proposition 3.2. Let $x$ be a variable of index 1. (Variables of index 2 are treated analogously.) Assume that for all $x_{1}<x$ we already know that $x_{1} \sigma$ is a ground term, i.e., an element of $T\left(\Sigma_{1} \cup \Sigma_{2}, \emptyset\right)$. Since $\sigma_{1}$ is a ground solution, we also know that $x \sigma_{1} \in T\left(\Sigma_{1} \cup X_{5,2}, \emptyset\right)$, and the elements of $X_{5,2}$ occurring in this term are smaller than $x$. Obviously, this implies that $x \sigma_{1} \sigma \in T\left(\Sigma_{1} \cup \Sigma_{2}, \emptyset\right)$.

Since $R$ is only an ordered rewriting system, this does not necessarily imply that $x \sigma=\left(x \sigma_{1} \sigma\right)_{\downarrow R}$ is a ground term as well. Rewriting steps with respect to $R$ may introduce variables from $Y$. For this reason we assume that the simplification ordering $\prec$ used during unfailing completion satisfies the property that at least one ground term is smaller than all variables in $Y$.

First, we show that this property can easily be satisfied. Let $z$ (of index i) be the least variable in $X_{4}$ with respect to the ordering $<$ that induces the linear constant restriction. Since $\sigma_{i}$ is a ground solution, $z \sigma_{i}$ is an element of $T\left(\Sigma_{i} \cup X_{5, j}, \emptyset\right)(i \neq j)$, and because $z$ is the least variable we even have $z \sigma_{i} \in$ $T\left(\Sigma_{i}, \emptyset\right)$. This shows that $\Sigma_{i}$ contains a constant symbol $c_{0}$. Obviously, there exist simplification orderings where $c_{0}$ is smaller than all elements of $Y$.

If the simplification ordering $\prec$ satisfies this property, then any term $t \in$ $T\left(\Sigma_{1} \cup \Sigma_{2}, \emptyset\right)$ has a normal form that is also in $T\left(\Sigma_{1} \cup \Sigma_{2}, \emptyset\right)$. This can be shown by induction on $\prec$. If $t$ is $R$-irreducible then $t$ is its own normal form. Otherwise, there exists a term $t_{1}$ such that $t \rightarrow_{R} t_{1}$. Assume that $t_{1}$ is not ground. We consider the term $t_{1}^{\prime}$ obtained from $t_{1}$ by replacing all variables by $c_{0}$. Since $c_{0}$ is smaller than all these variables, we know that $t \succ t_{1} \succ t_{1}^{\prime}$, and thus $t \rightarrow_{R} t_{1}^{\prime}$ is an admissible derivation. This shows that we can without loss of generality assume that $t_{1}$ is ground. But then we know by induction that its normal form $t_{1 \downarrow R}$ is ground. Since $R$ is confluent, $t_{1 \downarrow R}$ is also the normal form of $t$.

Conversely, $\Gamma_{0}$ may be ground solvable, even if the decomposition algorithm does not yield a pair of systems which are ground solvable.

Before giving an example where this situation occurs, let us explain why the proof of completeness given for the non-ground case cannot be adapted to 
the ground case. The reason is that a ground solution $\sigma$ of $\Gamma_{0}$ may substitute a variable of index $i$ by an $i$-term containing alien subterms. When $\sigma$ is transformed by projection to solutions $\sigma_{1}, \sigma_{2}$ of an output pair $\left(\Gamma_{5,1}, \Gamma_{5,2}\right)$ (see the proof of the "only if" part of Proposition 3.2), these alien subterms are replaced by variables. In general, for $\sigma_{i}$ not all of these variables are elements of $X_{5, j}, j \neq i$, i.e., not all of them are considered as constants in $\Gamma_{5, i}$. For this reason, $\sigma_{i}$ is not necessarily a ground solution of $\Gamma_{5, i}$.

Example 4.2 Let $\Sigma_{1}$ consist of the ternary function symbols $g$, the unary function symbol $f$, and the constant symbol $a$. Let $E_{1}=E_{1,1} \cup E_{1,2} \cup E_{1,3} \cup E_{1,4}$, where

$$
\begin{aligned}
& E_{1,1}=\{g(x, x, y)=a, g(x, y, x)=a, g(x, y, y)=a, g(x, a, y)=a\}, \\
& E_{1,2}=\{g(x, f(x), y)=a, g(x, f(y), y)=a, g(x, f(a), y)=a\}, \\
& E_{1,3}=\left\{g\left(x, g\left(y_{1}, y_{2}, y_{3}\right), z\right)=a\right\} \\
& E_{1,4}=\left\{g(x, f(f(y)), z)=a, g\left(x, f\left(g\left(y_{1}, y_{2}, y_{3}\right)\right), z\right)=a\right\} .
\end{aligned}
$$

Let $\Sigma_{2}$ consist of the two unary function symbols $h, k$ and the constant symbol $b$. For this signature we consider the theory

$$
E_{2}=\{h(h(x))=b, h(k(x))=b, h(b)=b\} .
$$

The disunification problem that we consider in the combined theory $E_{1} \cup E_{2}$ is

$$
\{g(b, y, h(y)) \neq a\} .
$$

It is easy to see that this problem has the ground solution $\{y \mapsto f(h(f(b)))\}$.

When we apply the decomposition algorithm we reach the system

$$
\Gamma_{2}=\{g(x, y, z) \neq a, x \doteq b, z \doteq h(y)\}
$$

after the first two steps. If, in the variable identification step, any of the variables $x, y, z$ are identified, the 1 -disequation $g(x, y, z) \neq a$ will not be solvable (because of the identities in $E_{1,1}$ ). This means that it is enough to consider the partition where $x, y, z$ are in separate classes.

Now let us consider different ways of choosing indices for $x, y, z$. Obviously, the 2-equations $x \doteq b$ and $z \doteq h(y)$ are only solvable if $x$ and $z$ get index 2 .

For $y$, let us first consider the case where ind $(y)=2$. The problem $\Gamma_{5,2}$ contains the equations $x \doteq b, z \doteq h(y)$ and the disequation $x \neq z$. Since $y$ is now treated as a variable in $\Gamma_{5,2}$, a ground solution $\sigma_{2}$ has to replaced it by a ground term $y \sigma_{2}$ built from the symbols $b, h, k$. Obviously, this implies $h\left(y \sigma_{2}\right)={ }_{E_{2}} b$, which shows that $\sigma_{1}$ cannot solve $\Gamma_{5,2}$.

Finally, let us consider the case where ind $(y)=1$. A ground solution $\sigma_{1}$ of

$$
\Gamma_{5,1}=\{g(x, y, z) \neq a, x \neq y, x \neq z, y \neq z\}
$$


can replace $y$ by a term $y \sigma_{1}$ that is built from the symbols $x, z, a, f, g$. Because of the disequations between variables, $y \sigma_{1}=x$ or $y \sigma_{1}=z$ is not possible. Also, $y \sigma_{1}=a$ is not possible because of the last identity in $E_{1,1}$. The identities in $E_{1,2}$ show that $y \sigma_{\mathrm{p}}$ cannot be one of $f(x), f(z), f(a)$. Because of the identity in $E_{1,3}$, the term $y \sigma_{1}$ cannot have top symbol $g$. The only remaining ground terms are of the form $f(f(t))$ or $f\left(g\left(t_{1}, t_{2}, t_{3}\right)\right)$, but these are prohibited by $E_{1,4}$.

In the example we have seen that $\Gamma_{0}$ has a ground solution, even though the decomposition algorithm does not yield systems $\Gamma_{5,1}, \Gamma_{5,2}$ that are ground solvable. The next conjecture could thus be that the systems $\Gamma_{5,1}, \Gamma_{5,2}$ have to be tested for solvability rather than ground solvability. But a closer look at the solutions $\sigma_{1}, \sigma_{2}$ one gets by projection from a ground solution $\sigma$ of $\Gamma_{0}$ reveals that these solutions satisfy an additional property: since $\sigma$ substitutes a variable $x$ of index $i$ by an $i$-term, $x \sigma_{i}$ is not a variable. In fact, it can easily be shown that $\sigma_{i}$ is a restrictive solution of $\Gamma_{5, i}$ (see Section 2 for the definition).

Lemma 4.3 Let $E_{1}$ and $E_{2}$ be two equational theories with disjoint signatures $\Sigma_{1}$ and $\Sigma_{2}$. Let $\Gamma_{0}$ be a disunification problem in $E_{1} \cup E_{2}$, i.e., an input problem for the decomposition algorithm. If $\Gamma_{0}$ has a ground solution $\sigma$, then there exists an output pair $\left(\Gamma_{5,1}, \Gamma_{5,2}\right)$ of the decomposition algorithm where each subsystem $\Gamma_{5, i}$ has a restrictive solution $\sigma_{i}$.

Proof. As above, let $R$ be an ordered-rewriting system obtained by applying unfailing completion to $E_{1} \cup E_{2}$. Without loss of generality we assume that there is a ground term that is smaller with respect to the simplification than all variables in $Y$. This makes sure that we can take an $R$-normalized ground solution of $\Gamma_{0}$. As in the proof of the "only if" part of Proposition 3.2, $\sigma$ determines the right choices in the nondeterministic decomposition steps. Then a bijection $\pi: T_{\downarrow R} \rightarrow Z$ (satisfying $\pi(x \sigma)=x$ for all $\left.x \in X_{4}\right)$ is used to define solutions $\sigma_{1}, \sigma_{2}$ of the obtained subsystems $\Gamma_{5,1}, \Gamma_{5,2}$ by projection. It remains to be shown that these solutions are restrictive.

Assume that for $x \in X_{5, i}$ we have $x \sigma_{i}={ }_{E_{i}} z$ for a variable $z \in Z$. Since $E_{i}$ is consistent this implies that $z$ occurs in $x \sigma_{i}$. In addition, we know that $x \sigma_{i}=(x \sigma)^{\pi_{i}}$, and that $x \sigma$ is an $i$-term that is $R$-irreducible and ground. For $z$ to occur in $x \sigma_{i}$ there must be an alien subterm $t$ of $x \sigma$ such that $\pi(t)=z$. Let $\tau$ denote the substitution

$$
\left\{y \mapsto s \mid \text { where } y \in Z \text { occurs in } x \sigma_{i} \text { and } \pi(s)=y\right\} .
$$

Now $x \sigma=x \sigma_{i} \tau={ }_{E_{i}} z \tau=t$, and thus $x \sigma={ }_{E} t$. Since $x \sigma$ and its subterm $t$ are $R$-irreducible, we get $x \sigma=t$. This is a contradiction since $x \sigma$ is an $i$-term and $t$ is a $j$-term $(i \neq j)$.

To get the opposite direction of the lemma, we need an additional restriction on the equational theories $E_{1}, E_{2}$ : the initial algebras have to be infinite. 
Lemma 4.4 Let $E_{1}, E_{2}$ be equational theories over disjoint signatures $\Sigma_{1}$ and $\Sigma_{2}$ such that $T\left(\Sigma_{i}, \emptyset\right) /=_{E_{\mathrm{i}}}$ is infinite for $i=1,2$. Let $\Gamma_{0}$ be a disunification problem in $E_{1} \cup E_{2}$, and suppose that, via decomposition, an output pair $\left(\Gamma_{5,1}, \Gamma_{5,2}\right)$ is reached such that each system $\Gamma_{5, i}$ has a restrictive solution. Then $\Gamma_{0}$ has a ground solution.

Proof. Let $\sigma_{i}$ be a restrictive solution of $\Gamma_{5, i}$ for $i=1,2$. We may assume that

$$
\begin{aligned}
& \sigma_{1}: X_{5,1} \rightarrow T\left(\Sigma_{1} \cup X_{5,2}, Y_{1}\right) \\
& \sigma_{2}: X_{5,2} \rightarrow T\left(\Sigma_{2} \cup X_{5,1}, Y_{2}\right)
\end{aligned}
$$

where the sets $Y_{1}$ and $Y_{2}$ are finite, disjoint and do not contain an element of $X_{4}=X_{5,1} \cup X_{5,2}$. Since $\sigma_{i}$ is restrictive we know that $x \sigma_{i} \neq E_{1}, y$ for all $r \in X_{5, i}$ and $y \in Y_{i}(i=1,2)$. Let us now consider the following extensions of the systems $\Gamma_{5, i}$ :

$$
\begin{aligned}
& \widehat{\Gamma}_{5,1}:=\Gamma_{5,1} \cup\left\{x \neq y ; x \in X_{5,1} \cup Y_{2}, y \in Y_{1} \cup Y_{2} \cup X_{5,2}, x \neq y\right\}, \\
& \widehat{\Gamma}_{5,2}:=\Gamma_{5,2} \cup\left\{x \neq y ; x \in X_{5,2} \cup Y_{1}, y \in Y_{1} \cup Y_{2} \cup X_{5,1}, x \neq y\right\} .
\end{aligned}
$$

The idea is to treat these systems as if they were a new output pair of the decomposition algorithm. For this purpose we choose a linear ordering which extends the linear ordering on $X_{4}$ from system $\Gamma_{4}$ and makes all elements $y \in$ $Y_{1} \cup Y_{2}$ smaller than the elements of $X_{4}$. We shall treat the elements $y \in Y_{i}$ as variables with index $j \neq i$. With this indexing and linear order, $\left(\hat{\Gamma}_{5,1}, \hat{\Gamma}_{5,2}\right)$ is in fact an output pair of the algorithm, corresponding to an input system $\widehat{\Gamma}_{0}$ which is an appropriate extension of $\Gamma_{0}$ by disequations.

In order to show that $\widehat{\Gamma}_{0}$ (and thus $\Gamma_{0}$ ) has a ground solution it suffices to prove (by Proposition 4.1) that each new subsystem $\widehat{\Gamma}_{5, i}$ has a ground solution $\widehat{\sigma}_{i}$. Without loss of generality, we shall restrict our attention to $\widehat{\Gamma}_{5,1}$. Note that the elements of $Y_{1}$ are treated as constants in $\hat{\Gamma}_{5.1}$. Let $Y_{2}$ be the set $\left\{y_{1} \ldots y_{n}\right\}$. and let $t_{1}, \ldots, t_{n} \in T\left(\Sigma_{1}, \emptyset\right)$ be pure 1-terms which are ground. and which are not equivalent modulo $E_{1}$ to each other and to any term $x \sigma_{1}$ for $x \in X_{5,1}$. Since $T\left(\Sigma_{1}, \emptyset\right) /=_{E_{1}}$ is infinite, we can be sure that such terms exist. We define

$$
\begin{aligned}
x \widehat{\sigma}_{1}:=x \sigma_{1} & \text { for } x \in X_{5,1}, \\
y_{i} \hat{\sigma}_{1}:=t_{i} & \text { for } i=1, \ldots, n .
\end{aligned}
$$

It is easy to see that $\hat{\sigma}_{1}$ is a ground solution of $\hat{\Gamma}_{5,1}$. In fact, since the elements of $Y_{1}$ are now treated as constants, it is a ground substitution, and it obviously solves the equations and disequations of $I_{5,1}$. Restrictiveness of $\sigma_{1}$ guarantees that $\widehat{\sigma}_{1}$ solves the disequations $x \neq y$ for $x \in X_{5,1}$ and $y \in Y_{1}$; the choice of the $t_{j}$ guarantees that $\hat{\sigma}_{1}$ solves the disequations $x \neq y$ for $x \in X_{5,1} \cup Y_{2}$ and $x \neq y \in Y_{2}$. Finally, for $x \in X_{5,2}$ and $y \in Y_{2}$, we have $r \widehat{\sigma}_{1}=x \neq F_{1} y \widehat{\sigma}_{1} \in T\left(\Sigma_{1}, \emptyset\right)$ since $E_{1}$ is consistent. 
It should be noted that restrictiveness of the solutions of $\Gamma_{5,1}, \Gamma_{5,2}$ is necessary for the lemma to hold. This can be demonstrated by giving an example of theories $E_{1}, E_{2}$ for which

- the initial algebras are infinite, and

- there exists a disunification problem $\Gamma_{0}$ for $E_{1} \cup E_{2}$ which is solvable, but not ground solvable.

In fact, if $\Gamma_{0}$ is solvable, Proposition 3.2 implies that the decomposition algorithm yields a pair $\Gamma_{5,1}, \Gamma_{5,2}$ that is solvable as well. Since $\Gamma_{0}$ is not ground solvable this shows that just assuming solvability of $\Gamma_{5,1}, \Gamma_{5,2}$ is not enough to get ground solvability of $\Gamma_{0}$. Because of Lemma 4.4 this also means that one of the systems $\Gamma_{5,1}, \Gamma_{5,2}$ has a solution but no restrictive solution.

Example 4.5 Let $\Sigma_{1}$ consist of the ternary function symbol $g$, the unary function symbol $f$, and the constant symbol $a$. We consider the theory

$$
\begin{gathered}
E_{1}=\left\{g(x, f(y), z)=a, g\left(x, g\left(y_{1}, y_{2}, y_{3}\right), z\right)=a,\right. \\
g(x, a, z)=a, g(x, y, x)=a\} .
\end{gathered}
$$

The signature $\Sigma_{2}$ consists of the two unary function symbols $h, k$ and the constant symbol $b$. As our second theory we take

$$
E_{2}=\{h(h(x))=b, h(k(x))=b, h(b)=b\} .
$$

The initial algebra of $E_{1}$ is infinite since the infinitely many terms of the form $f(f(\cdots f(a) \cdots))$ are not equivalent modulo $E_{1}$. For $E_{2}$, we have infinitely many non-equivalent terms of the form $k(k(\cdots k(b) \cdots))$.

Now consider the disunification problem $\Gamma_{0}=\{g(b, x, h(x)) \neq a\}$. Obviously, the identity substitution is a solution of $\Gamma_{0}$. Now assume that $\sigma$ is an arbitrary solution of $\Gamma_{0}$. Because of the first three identities in $E_{1}$ we know that $x \sigma$ cannot be a 1-term. On the other hand, $x \sigma$ cannot be a 2 -term either. In fact, the identities in $E_{2}$ would imply that $h(x \sigma)=_{E} b$, and thus the fourth identity in $E_{1}$ would imply that $g(b, x \sigma, h(x \sigma))=E_{E} a$. Hence $x \sigma$ must be a variable, which shows that $\sigma$ cannot be a ground solution of $\Gamma_{0}$.

Obviously, Lemma 4.3 and 4.4 can also be shown for the combination of more than two theories. Thus we obtain the main theorem of this section.

Theorem 4.6 Let $E_{i}, i=1, \ldots, n$, be equational theories over disjoint signatures $\Sigma_{i}$, and suppose that the initial algebras $T\left(\Sigma_{i}, \emptyset\right) /=_{E_{i}}$ are infinite. If restrictive solvability of $E_{i}$-disunification problems with linear constant restriction is decidable for $i=1, \ldots, n$, then ground solvability of disunification problems is decidable for $E_{1} \cup \ldots \cup E_{n}$. 
If one of the theories, say $E_{n}$, satisfies a stronger restriction, the condition that the initial algebras are infinite can be dropped for the other theories.

Corollary 4.7 Let $E_{1}, \ldots, E_{n}$ be equational theories over disjoint signatures $\Sigma_{1}$, $\ldots, \Sigma_{n}$. Assume that $T\left(\Sigma_{n}, \emptyset\right) /=_{E_{n}}$ is infinite, and that every solvable $E_{n}$-disunification problem with linear constant restriction has a ground solution. Then ground solvability of disunification problems in $E_{1} \cup \ldots \cup E_{n}$ is decidable if restrictive solvability of $E_{i}$-disunification problems with linear constant restriction is decidable for $i=1, \ldots, n$.

This can be seen by an inspection of the proof of Lemma 4.4. Since we know that a solvable system $\Gamma_{5, n}$ has a ground solution, we do not need any alien $i$ terms $(i \neq n)$ to get rid of variables in solutions of $\Gamma_{5, n}$. For the other theories, the assumption that $T\left(\Sigma_{n}, \emptyset\right) /=_{E_{n}}$ is infinite provides for the required alien terms.

An important case to which this corollary applies is the combination with a free theory. We call an equational theory $F$ the free theory with signature $\Sigma$ iff $\operatorname{sig}(F)=\Sigma$ and $=_{F}$ is just the syntactic equality of terms. Obviously, considering elementary disunification in the combination of a theory $E$ with a free theory corresponds to considering general disunification for $E$.

Corollary 4.8 Let $\Sigma_{1}, \ldots, \Sigma_{n}$ be disjoint signatures, $E_{1}, \ldots, E_{n-1}$ be equational theories over $\Sigma_{1}, \ldots, \Sigma_{n-1}$, and let $E_{n}$ be the free theory with signature $\Sigma_{n}$. Assume that $\Sigma_{n}$ contains at least one function symbol of arity greater zero and one constant. Then ground solvability of disunification problems in $E_{1} \cup \ldots \cup E_{n}$ is decidable if restrictive solvability of $E_{i}$-disunification problems with linear constant restriction is decidable for $i=1, \ldots, n-1$.

Proof. Obviously, the condition that there is at least one constant and one (non-constant) function symbol in $\Sigma_{n}$ implies that $T\left(\Sigma_{n}, \emptyset\right) /=_{E_{n}}=T\left(\Sigma_{n}, \emptyset\right)$ is infinite.

Now we show that an $E_{n}$-disunification problem with linear constant restriction, say $(\Gamma, X, C,<)$, is solvable iff it is ground solvable. Obviously, ground solvability implies solvability. Thus assume that $\sigma$ is a (not necessarily ground) solution of $(\Gamma, X, C,<)$. Assume that $y_{1}$ is a variable occurring in $x \sigma$ for some $x \in X$. Let $t \in T\left(\Sigma_{n}, \emptyset\right)$ be a term that is larger than all the terms $x^{\prime} \sigma$ for $x^{\prime} \in X$. We define $\sigma^{\prime}:=\sigma \circ\left\{y_{1} \mapsto t\right\}$. Obviously, $\sigma^{\prime}$ solves the equations in $\Gamma$. It satisfies the constant restriction since $t$ does not introduce elements of $C$. Finally, consider a disequation $s_{1} \neq s_{2}$ of $\Gamma$. We know that $s_{1} \sigma \neq s_{2} \sigma$. Thus there exists an occurrence where these two terms disagree. Let the terms at this occurrence be $t_{1}, t_{2}$. If $t_{1}$ and $t_{2}$ are non-variable terms they have different top symbol. Hence we will still get a disagreement after applying $\left\{y_{1} \mapsto t\right\}$. The same is true if one is a variable different from $y_{1}$. Thus assume that $t_{1}=y_{1}$. If $y_{1}$ does not occur in 
$t_{2}$ then $y_{1}\left\{y_{1} \mapsto t\right\}=t \neq t_{2}=t_{2}\left\{y_{1} \mapsto t\right\}$ (since $t$ is larger than $t_{2}$ by choice of $t$ ). Otherwise, $y_{1}\left\{y_{1} \mapsto t\right\}=t$ is a strict subterm of $t_{2}\left\{y_{1} \mapsto t\right\}$. Thus we have seen that $s_{1} \sigma^{\prime} \neq s_{2} \sigma^{\prime}$. Using this method we can successively eliminate all variables in the image of $\sigma$.

To apply Corollary 4.7, it remains to be shown that restrictive solvability of $E_{n}$-disunification problems with linear constant restriction is decidable. We have just seen that solvability is equivalent to ground solvability. Since ground solutions are always restrictive it is thus enough to show that solvability of $E_{n^{-}}$ disunification problems $\Gamma$ with linear constant restriction is decidable. This has already been shown in the proof of Corollary 3.3.

\section{Applications of the Method}

The methods developed in the preceding two sections will now be applied to the combination of $A, A C, A C I$, and free theories. An equational theory is called an $A$-theory iff its signature consists of a binary function symbol $h$, and it contains the single axiom $h(h(x, y), z)=h(x, h(y, z))$ (associativity). For $A C$-theories, one has an additional axiom $h(x, y)=h(y, x)$ (commutativity), and for $A C I$-theories there is a third axiom $h(x, x)=x$ (idempotence).

Theorem 5.1 Solvability of disunification problems is decidable for every theory which is a disjoint combination of finitely many A-, AC-, and ACI-theories and a free theory. To get decidability of ground solvability by our method we have to assume that the free theory contains at least one constant symbol and one function symbol of arity greater than 0 .

Since existential equational formulae can be seen as disjunction of disunification problems we have the following immediate consequence of the theorem.

Corollary 5.2 Let $\Sigma$ be a signature consisting of $n \geq 1$ binary function symbols $h_{1}, \ldots, h_{n}$, and at least one constant and one additional non-constant function symbol. Let $A_{n}, A C_{n}$, and $A C I_{n}$ respectively stand for associativity, associativity and commutativity, and associativity, commutativity and idempotence of the function symbols $h_{i}$.

1. The existential theories of the free algebra $T(\Sigma, Y) /=_{A_{n}}$ and the initial algebra $T(\Sigma, \emptyset) /={ }_{A_{n}}$ are decidable.

2. The existential theories of the free algebra $T(\Sigma, Y) /={ }_{A C_{n}}\left(T(\Sigma, Y) /={ }_{A C I_{n}}\right)$, and the initial algebra $T(\Sigma, \emptyset) /={ }_{A C_{n}}\left(T(\Sigma, \emptyset) /=_{A C I_{n}}\right)$ are NP-decidable. 
For $A C$, decidability has already been shown by Comon [Com88]. The result for $A$ seems to be new. There is no real hope to extend these decidability results to equational formulae with more complex quantifier prefix. A recent result by Treinen [Tr92] shows that already the $\Sigma_{2}$ fragment $^{3}$ of the theory of the ground term algebra modulo $A$ is undecidable. For $A C$, Treinen shows that the $\Sigma_{3^{-}}$ fragment is undecidable, both for the free algebra and the initial algebra.

To prove Theorem 5.1, it remains to be shown that solvability and restrictive solvability of disunification problems with linear constant restriction are decidable for $A$-theories, and NP-decidable for $A C$-, $A C I$ - and free theories. Decidability for free theories has already been shown in the proof of Corollary 4.8. Obviously, this decision method is of polynomial time complexity. We shall consider $A, A C$ and $A C I$ in the following.

Firstly, it turns out that restrictiveness of a solution is here not a real constraint. In fact, we shall show for these theories that a disunification problem with linear constant restriction has a solution iff it has a restrictive solution. In particular, this means that solvability and ground solvability for the combined theory are equivalent.

Proposition 5.3 Let $E$ be an A-, AC-, or ACI-theory. Then solvability and restrictive solvability of $E$-disunification problems with linear constant restriction. are equivalent.

Proof. For the three types of theories it is easy to see that any term containing a subterm of the form $h\left(y_{1}, y_{2}\right)$ (for distinct variables $\left.y_{1}, y_{2}\right)$ cannot be equivalent to a variable. Let $(\Gamma, X, C,<)$ be an $E$-unification problem with linear constant restriction, and let $\sigma$ be a solution of $\Gamma$. Now assume that $x \sigma={ }_{E} y$ for $x \in X$ and a variable $y$, which means that $\sigma$ is not restrictive.

We take distinct variables $y_{1}, y_{2}$ that do not occur in $z \sigma$ for any variable $z$ in $\Gamma$, and define $\sigma^{\prime}:=\sigma \circ\left\{y \mapsto h\left(y_{1}, y_{2}\right)\right\}$. Obviously, $\sigma^{\prime}$ solves the equations in $\Gamma$, and it still satisfies the constant restriction. In addition, we have $x \sigma^{\prime}={ }_{E} h\left(y_{1}, y_{2}\right)$, which shows that $x \sigma^{\prime}$ is no longer equivalent to a variable. It remains to be shown that $\sigma^{\prime}$ also solves the disequations of $\Gamma$. Let $s \neq t$ be such a disequation. We know that $s \sigma \neq_{E} t \sigma$.

(1) First, we consider the case where $E$ is an $A$-theory. Since we are working modulo associativity, terms can be seen as words over the alphabet $\Sigma$ of constant and variable symbols. We know that the two words $s \sigma, t \sigma$ are different. First, assume that one is a strict prefix of the other. Obviously, this means that the same holds for the words associated with $s \sigma^{\prime}$ and $t \sigma^{\prime}{ }^{4}$ Otherwise (i.e., if none is

\footnotetext{
${ }^{3}$ consisting of the closed formulae with quantifier prefix of the form $\exists \vec{x} \forall \vec{y}$

${ }^{4}$ Note that we do not have a unit element for $h$, which implies that any variable has to be replaced by a nonempty word.
} 
a prefix of the other) there exist words $u, v, w$ and distinct elements $a, b$ of the alphabet $\Sigma$ such that $s \sigma=u a v$ and $t \sigma=u b w$. The words $s \sigma^{\prime}$ and $t \sigma^{\prime}$ have the same prefix $u\left\{y \mapsto y_{1} y_{2}\right\}$. The next symbols are either $a$ and $b$ (if none is equal to $y$ ), or $y_{1}$ and one of $a$ and $b$. Both cases yield that the two words $s \sigma^{\prime}$ and $t \sigma^{\prime}$ are different.

(2) Now consider the case where $E$ is an $A C$-theory. We can associate with each term $r$ a mapping $\alpha_{r}$ from the alphabet $\Sigma$ of variables and constants to the nonnegative integers as follows:

$$
\alpha_{r}(a) \text { is the number of occurrences of } a \text { in } r \text {. }
$$

It is easy to see that we have $r_{1}={ }_{E} r_{2}$ iff $\alpha_{r_{1}}$ and $\alpha_{r_{2}}$ are the same function, i.e., if for all $a \in \Sigma$ one has $\alpha_{r_{1}}(a)=\alpha_{r_{2}}(a)$. Thus we know $\alpha_{s \sigma} \neq \alpha_{t \sigma}$. Evidently, $\alpha_{s \sigma^{\prime}}(a)=\alpha_{s \sigma}(a)$ for all variables or constants $a \notin\left\{y, y_{1}, y_{2}\right\}$. In addition, $\alpha_{s \sigma}\left(y_{i}\right)=0$, and $\alpha_{s \sigma^{\prime}}\left(y_{i}\right)=\alpha_{s \sigma}(y)(i=1,2)$. The same holds for $t$ in place of $s$. Thus $\alpha_{s \sigma} \neq \alpha_{t \sigma}$ implies $\alpha_{s \sigma^{\prime}} \neq \alpha_{t \sigma^{\prime}}$. This shows that $s \sigma^{\prime} \neq_{E} t \sigma^{\prime}$.

(3) The cases where $E$ is an $A C I$-theory can be handled similarly. Instead of the invariant $\alpha_{r}$ we just take the mapping $\beta_{r}$, which is defined as follows:

$$
\beta_{r}(a)= \begin{cases}1 & \text { if } a \text { occurs in } r \\ 0 & \text { otherwise }\end{cases}
$$

To sum up, we have seen that in each case $\sigma^{\prime}$ also solves the disequations in $\Gamma$. By successively applying this method to all variables $x \in X$ for which $x \sigma$ is equivalent to a variable, we can construct a restrictive solution of $\Gamma$.

Secondly, it can be shown that solvability of disunification problems with linear constant restriction for $A, A C$ and $A C I$ can be reduced to ground solvability over an appropriately enlarged signature.

Proposition 5.4 Let $E$ be an A-, AC-, or ACI-theory. An E-disunification problem with linear constant restriction $(\Gamma, X, C,<)$ containing $m$ disequations has a solution if and only if it has a ground solution in the initial algebra $T(\{h\} \cup$ $C \cup D, \emptyset) /=_{E}$, where $D=\left\{d_{1}, \ldots, d_{2 m+1}\right\}$ is a set of $2 m+1$ constants that is disjoint to $C$.

Proof. In the previous proof we have used that terms $r_{1}, r_{2}$ that are different modulo $E$ yield "disagreement symbols" that are responsible for this difference.

If $E$ is an $A$-theory, we get the disagreement symbols $a, b$ if $r_{1}$ is the word $u a v$ and $r_{2}$ is the word $u b w$ (where $a, b$ are distinct symbols from the alphabet of variables and constants). The other case, where one word is a strict prefix of the other, does not yield a disagreement symbol. But in this second case, the words $r_{1} \tau$ and $r_{2} \tau$ will be different for arbitrary substitutions $\tau$. 
If $E$ is an $A C$-theory ( $A C I$-theory) we take as disagreement symbol the symbol $a$ for which $\alpha_{r_{1}}(a) \neq \alpha_{r_{2}}(a)\left(\beta_{r_{1}}(a) \neq \beta_{r_{2}}(a)\right)$.

Now let $\sigma$ be a solution of $\Gamma$, and let $\left\{y_{1}, \ldots, y_{k}\right\}$ be all variables that are disagreement symbols of $s \sigma, t \sigma$ for some disequation $s \neq t$ in $\Gamma$. Since a disequation can yield at most two disagreement symbols, we have $k \leq 2 m$. Let $\tau$ be the substitution

$$
\begin{gathered}
\left\{y_{i} \mapsto d_{i} \mid i=1, \ldots, k\right\} \cup \\
\left\{y \mapsto d_{k+1} \mid y \notin\left\{y_{1}, \ldots, y_{k}\right\} \text { occurs in } x \sigma \text { for some } x \in X\right\} .
\end{gathered}
$$

Obviously, $\sigma \circ \tau$ is a ground solution of the equations in $\Gamma$, and it satisfies the constant restriction (since occurrence of the elements in $D$ is not constrained). It solves the disequations since disagreement symbols are mapped to distinct new constants.

Thus, eventually one has to consider ground solvability of disunification problems with linear constant restriction for $A, A C$ and $A C I$. This is done in the following three subsections.

Before that, let us mention that the method of reducing solvability to ground solvability described above does not work for arbitrary theories. In fact, in Proposition 5.4 the number of disequations could be used to determine the number of constants to be added. In the general case, we know that whenever a disunification problem is solvable it is ground solvable over a signature appropriately enlarged by finitely many constant symbols. (Just treat the variables in a solution as constants.) But how many constants have to be added may also depend on the structure of the equations and disequations, and not just on the number of disequations.

An example of a theory where this is the case is the theory

$$
A I=\{h(h(x, y), z)=h(x, h(y, z)), h(x, x)=x\} .
$$

For $n \geq 1$, let $X_{n}$ denote a set consisting of $n$ different variables. From the results in [Fen71] one can easily deduce that for each $n \geq 1$ there exist terms $s_{n}, t_{n} \in T\left(\{h\}, X_{n+2}\right)$ such that

- $s_{n} \neq \neq_{A I} t_{n}$, but

- $s_{n} \sigma={ }_{A I} t_{n} \sigma$ for any substitution $\sigma$ that replaces the variables in $s_{n}, t_{n}$ by terms in $T\left(\{h\}, X_{n}\right)$.

This means that the $A I$-disunification problem $\Gamma=\left\{s_{n} \neq t_{n}\right\}$ is solvable (by the identity substitution), but it is not ground solvable if one has at most $n$ constants.

It is not clear how to determine the appropriate number of constants for an arbitrary $A I$-disunification problem. For this reason, deciding solvability of $A I$ disunification problems is still an open problem, even though ground solvability 
for a finite number of constants is trivially decidable (since finitely generated AI-free algebras are finite).

\subsection{Ground Solvability for $A$}

For an $A$-theory $E$ one can use a method described by Büchi and Senger [BüS86] to reduce ground solvability of $E$-disunification problems with linear constant restriction to ground solvability of $E$-unification problems with linear constant restriction. Solvability ${ }^{5}$ of unification problems with linear constant restriction for $A$-theories is treated in [BS91b].

For the reader's convenience we shall briefly describe the argument in [B̈̈S86]. As mentioned above, terms modulo an $A$-theory may be considered as words over the alphabet of variables and constants. Assume that $C$ is the set of constants available for building ground solutions. Büchi and Senger show that a disequation can be expressed by a positive boolean combination of equations. i.e., a combination not involving negation.

To define this formula, we have to introduce two abbreviations. For two words $w_{1}, w_{2}$ the expression $w_{1} \triangleleft w_{2}$ stands for the equation $w_{1} x=w_{2}$, and $w_{1} \unlhd w_{2}$ stands for the disjunction of equations $w_{1}=w_{2} \vee w_{1} x=w_{2}$. Here $x$ is meant to be a variable different from the ones occurring in our disunification problem, and we assume that different expressions of this form use different variables.

A disequation $w_{1} \neq w_{2}$ is equivalent to the formula

$$
\begin{gathered}
w_{1} \triangleleft w_{2} \vee w_{2} \triangleleft w_{1} \vee \\
\bigvee_{a \neq b \in C}\left(\left(z a \unlhd w_{1}\right) \wedge\left(z b \unlhd w_{2}\right)\right) \vee \\
\bigvee_{a \neq b \in C}\left(\left(a \unlhd w_{1}\right) \wedge\left(b \unlhd w_{2}\right)\right) .
\end{gathered}
$$

Here $z$ is also meant to be a new variable. Obviously, this formula just expresses the fact that two words are different iff one is a strict prefix of the other, or there are disagreement symbols $a, b$. Since we want to have a ground solution, these disagreement symbols must come from the set of available constant symbols.

Since we can bring any Boolean combination of equations into disjunctive normal form, this shows that ground solvability of an $E$-disunification problem with linear constant restriction can be reduced to testing solvability of a finite number of $E$-unification problems with linear constant restriction.

\footnotetext{
${ }^{5}$ Recall that, for unification, solvability and ground solvability are equivalent.
} 


\subsection{Ground Solvability for $A C$}

For $A C$, ground solvability of a disunification problem with linear constant restriction can be reduced to an integer programming problem (of a size that is polynomial in the size of the original problem). The integer programming problem can then be solved by one of the known NP-algorithms (see, e.g., [Sc86], pp.239). Instead of giving a formal definition of this reduction for the general case, we illustrate it by an example.

Let $E$ be an $A C$-theory for the binary function symbol $h$. We consider the $E$-disunification problem

$$
\begin{aligned}
\Gamma=\{h(x, h(x, h(c, h(c, c)))) & \doteq h(y, h(y, h(y, h(y, b)))), \\
h(x, h(x, h(x, h(y, y)))) & \doteq h(x, c), \\
h(x, h(y, h(y, h(y, y)))) & \neq h(x, h(x, h(c, h(y, x))))\}
\end{aligned}
$$

with the constant restriction induced by $c<x<b<y$. Assume that we want to decide ground solvability over the alphabet of constants $\{b, c, d, e\}$.

For each of these constants, we introduce a system of linear equations. These systems will correspond to the equations in $\Gamma$. The variables occurring in the linear equations stand for the number of occurrences of the respective constant in the image of $x$ and $y$, respectively, of possible solutions of $\Gamma$. The coefficients of these variables in the equations are the number of occurrences of $x$ and $y$, respectively, in $\Gamma$. Thus we get the four systems

$$
\begin{aligned}
\left(\begin{array}{ll}
2 & 0 \\
3 & 2
\end{array}\right)\left(\begin{array}{l}
x_{b} \\
y_{b}
\end{array}\right) & =\left(\begin{array}{ll}
0 & 4 \\
1 & 0
\end{array}\right)\left(\begin{array}{l}
x_{b} \\
y_{b}
\end{array}\right)+\left(\begin{array}{l}
1 \\
0
\end{array}\right) \\
\left(\begin{array}{ll}
2 & 0 \\
3 & 2
\end{array}\right)\left(\begin{array}{l}
x_{c} \\
y_{c}
\end{array}\right)+\left(\begin{array}{l}
3 \\
0
\end{array}\right) & =\left(\begin{array}{ll}
0 & 4 \\
1 & 0
\end{array}\right)\left(\begin{array}{l}
x_{c} \\
y_{c}
\end{array}\right)+\left(\begin{array}{l}
0 \\
1
\end{array}\right) \\
\left(\begin{array}{ll}
2 & 0 \\
3 & 2
\end{array}\right)\left(\begin{array}{l}
x_{d} \\
y_{d}
\end{array}\right) & =\left(\begin{array}{ll}
0 & 4 \\
1 & 0
\end{array}\right)\left(\begin{array}{l}
x_{d} \\
y_{d}
\end{array}\right) \\
\left(\begin{array}{ll}
2 & 0 \\
3 & 2
\end{array}\right)\left(\begin{array}{l}
x_{e} \\
y_{e}
\end{array}\right) & =\left(\begin{array}{ll}
0 & 4 \\
1 & 0
\end{array}\right)\left(\begin{array}{l}
x_{e} \\
y_{e}
\end{array}\right) .
\end{aligned}
$$

In addition, since we do not have a unit element for $h$, the variables $x, y$ have to be substituted by nonempty terms. This is expressed by the inequalities

$$
x_{b}+x_{c}+x_{d}+x_{e}>0 \text { and } y_{b}+y_{c}+y_{d}+y_{e}>0 .
$$

It should be obvious how to express the constant restriction with the help of some additional equations: If a constant must not occur in the image of a variable, the corresponding variable in the system of linear equations has to be zero. In our example, we get the additional equation

$$
x_{b}=0
$$


because $x<b$ means that $b$ must not occur in the image of $x$.

The disequation

$$
h(x, h(y, h(y, h(y, y)))) \neq h(x, h(x, h(c, h(y, x))))
$$

is translated into a disjunction of four negated equations

$$
\begin{aligned}
& \left(x_{b}+4 y_{b} \neq 3 x_{b}+y_{b}\right) \vee\left(x_{c}+4 y_{c} \neq 3 x_{c}+y_{c}+1\right) \vee \\
& \left(x_{d}+4 y_{d} \neq 3 x_{d}+y_{d}\right) \vee\left(x_{e}+4 y_{e} \neq 3 x_{e}+y_{e}\right) .
\end{aligned}
$$

A nondeterministic algorithm for ground solvability first chooses one of the disjuncts for each disequation. After this nondeterministic step one has a system of linear diophantine equations and inequations (a negated equation can be scen as two inequations). As mentioned at the beginning of this subsection, solvability of such a problem can be decided by an NP-algorithm.

\subsection{Ground Solvability for $A C I$}

Finally, for an $A C I$-theory $E$, ground solvability of an $E$-disunification problem with linear constant restriction can be reduced to satisfiability of Boolean formulae. This problem is again NP-decidable (sce, e.g., [Coo71]).

Let $\Gamma$ be an $E$-disunification problem with linear constant restriction, let $X$ be the variables occurring in $\Gamma$, and assume that we are looking for a ground solution using only constants from the finite set $C$. For each pair $\langle c, x\rangle \in C \times X$ we introduce a propositional variable $p_{c, x}$, with the intended reading " $c$ occurs in the image of $x "$.

For a term $s \in T(X, C)$, let $X_{s}$ denote the set of variables occurring in $s$, and $C_{s}$ the set of constants occurring in $s$. With each equation $s \doteq t \in \Gamma$ we associate a Boolean formula $\Phi(s, t)$ that is defined as

$$
\begin{gathered}
\bigwedge_{c \in C_{s} \backslash C_{t} x \in X_{t}} \bigvee_{c, x} p_{c, x} \wedge \bigwedge_{c \in C_{t} \backslash C_{s}} \bigvee_{x \in X_{s}} p_{c, x} \wedge \\
\bigwedge_{c \in C \backslash\left(C_{s} \cup C_{t}\right)}\left(\bigvee_{x \in X_{s}} p_{c, x} \Leftrightarrow \bigvee_{y \in X_{t}} p_{c, y}\right) .
\end{gathered}
$$

The first part of the formula says that each constant $c$ that occurs on the left hand side of the equation, but not on the right hand side, must be introduced by some variable of the right hand side. Accordingly, this has to be true for constants occurring only on the right hand side. For constants occurring on both sides we have no restriction. Finally, constants that do not occur on either side of the equation can be introduced on the left hand side iff they are introduced on the right hand side. 
With each disequation $s \neq t$ we associate the formula $\neg \Phi(s, t)$. The fact that any variable $x \in X$ must be replaced by a nonempty ground term is expressed by the formula

$$
\bigwedge_{x \in X} \bigvee_{c \in C} p_{c, x}
$$

The treatment of constant restrictions is also very simple: for $x<c$ we just add a formula $\neg p_{c, x}$.

If we take the conjunction of all these formulae we get a Boolean formula of a size that is polynomial in the size of our original problem $\Gamma$. It is easy to see that this formula is satisfiable iff $\Gamma$ has a ground solution.

\section{Conclusion}

Since constraint approaches to theorem proving, term rewriting, and logic programming are gaining in importance, constraint solving has become a major research issue in these areas. An important subproblem is the question of how to combine different constraint solving techniques. The present paper can be seen as a contribution to this field, where the constraints are existentially quantified equational formulae that have to be solved in the initial or the free algebra modulo an equational theory. We have seen that the methods developed for the combination of unification algorithms can be applied for disunification as well. For solvability of disunification problems, this was relatively straightforward, even though the proofs became more involved. For ground solvability we surprisingly have to consider a restricted type of solvability (instead of ground solvability) in the single theories.

For the theories $A, A C$ and $A C I$, solvability and restrictive solvability coincide, which implies that solvability and ground solvability in their combination with a non-trivial free theory are equivalent. However, we have given an example of a theory where solvability does not imply restrictive solvability (see Example 4.5). An interesting open problem is under what conditions solvability and restrictive solvability coincide, and when solvability and ground solvability refer to the same problem.

\section{References}

[BS91a] F. Baader, K.U. Schulz, "Unification in the Union of Disjoint Equational Theories: Combining Decision Procedures," DFKI-Research Report RR-91-33; also in Proceedings of the 11th International Conference on Automated Deduction, LNCS subseries on AI 607 (1992), pp.50-65. 
[BS91b] F. Baader, K.U. Schulz, "General A- and AX-Unification via Optimized Combination Procedures," CIS-Report 92-58, CIS, University Munich; also to appear in the Proceedings of the Second Workshop on Word Equations and Related Topics IWWERT '91, Rouen 1991, LNCS.

[Bür88] H.J. Bürckert, "Solving Disequations in Equational Theories," Proceedings of the 9th International Conference on Automated Deduction, Argonne, LNCS 310, Springer 1988.

[Bür90] H.J. Bürckert, "A Resolution Principle for Clauses with Constraints," Proceedings of the 10th International Conference on Automated Deduction, LNCS 449, 1990.

[BüS86] J.R. Büchi, S. Senger, "Coding in the Existential Theory of Concatenation," Arch. math. Logik 26 (1986/7), pp.101-106.

[Col84] A. Colmeraner, "Equations and Inequations on Finite and Infinite Trees," In Proceedings FGCS'84, pp.85-99.

[Col90] A. Colmerauer, "An Introduction to PROLOG III," C. ACM 33, 1990.

[Com88] H. Comon, "Unification et Disunification. Théorie et Applications," PhD Thesis, Institut National Polytechnique de Grenoble, Grenoble, France, 1988.

[Com91] H. Comon, "Disunification: a Survey," in J.-L. Lassez, G. Plotkin (editors), Computational Logic, MIT Press, 1991.

[Coo71] S.A. Cook, "The Complexity of Theorem-Proving Procedures," Proc. Third Annual ACM Symposium on the Theory of Computing, Association for Computing Machinery, New York 1971, pp.151-158.

[DJ89] N. Dershowitz, J.P. Jouannaud, "Rewrite Systems," Unité Associée au CNRS UA 410: AL KHOWARIZMI, Rapport de Recherche $n^{\circ} 478$, 1989 ; also in Volume B of "Handbook of Theoretical Computer Science," North-Holland 1990, pp.244-320.

[Fen71] C.F. Fennemore, "All Varieties of Bands I, II," Math. Nachr. 48, 1971, pp.237-262.

[JK91] J.P. Jouannaud, C. Kirchner, "Solving Equations in Abstract Algebras: A Rule-Based Survey of Unification," in J.-L. Lassez, G. Plotkin (editors), Computational Logic, MIT Press, 1991.

[JL87] J. Jaffar, J.L. Lassez, "Constraint Logic Programming," Proceedings of 14th POPL Conference, Munich, 1987. 
[KN91] D. Kapur, P. Narendran, "Complexity of Unification Problems with Associative-Commutative Operators," J. Automated Reasoning 9, 1992, pp.261-288.

[KK89] C. Kirchner, H. Kirchner, "Constrained Equational Reasoning," Proceedings of SIGSAM 1989 International Symposium on Symbolic and Algebraic Computation, ACM Press, 1989.

[SS89] M. Schmidt-Schauß, "Combination of Unification Algorithms," J. Symbolic Computation 8, 1989.

[Sc86] A. Schrijver, "Theory of Linear and Integer Programming," John Wiley \& Sons, Chichester 1986.

[Tr92] R. Treinen, "A New Method for Undecidability Proofs of First Order Theories," J. Symbolic Computation 14, 1992, pp.437-457. 


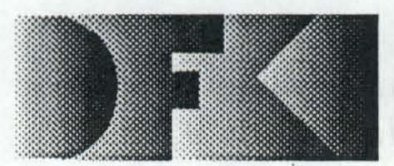

Deutsches

Forschungszentrum

für Künstliche

Intelligenz $\mathrm{GmbH}$
DFKI

-Bibliothek-

PF 2080

D-6750 Kaiserslautern

FRG

\section{DFKI Publikationen}

Die folgenden DFKI Veröffentlichungen sowie die aktuelle Liste von allen bisher erschienenen Publikationen können von der oben angegebenen Adresse bezogen werden.

Die Berichte werden, wenn nicht anders gekennzeichnet, kostenlos abgegeben.

\section{DFKI Research Reports}

RR-92-10

$M$. Bauer: An Interval-based Temporal Logic in a Multivalued Setting

17 pages

\section{RR-92-11}

Susane Biundo, Dietmar Dengler, Jana Koehler:

Deductive Planning and Plan Reuse in a

Command Language Environment

13 pages

\section{RR-92-13}

Markus A. Thies, Frank Berger:

Planbasierte graphische Hilfe in

objektorientierten Benutzungsoberflächen

13 Seiten

RR-92-14

Intelligent User Support in Graphical User Interfaces:

1. InCome: A System to Navigate through Interactions and Plans

Thomas Fehrle, Markus A. Thies

2. Plan-Based Graphical Help in ObjectOriented User Interfaces

22 pages

Markus A. Thies, Frank Berger

\section{RR-92-15}

Winfried Graf: Constraint-Based Graphical

Layout of Multimodal Presentations

23 pages

\section{RR-92-16}

Jochen Heinsohn, Daniel Kudenko, Berhard Nebel, Hans-Jürgen Profitlich: An Empirical Analysis of Terminological Representation Systems 38 pages

\section{DFKI Publications}

The following DFKI publications or the list of all published papers so far can be ordered from the above address.

The reports are distributed free of charge except if otherwise indicated.

\section{RR-92-17}

Hassan Aït-Kaci, Andreas Podelski, Gert Smolka:

A Feature-based Constraint System for Logic

Programming with Entailment

23 pages

RR-92-18

John Nerbonne: Constraint-Based Semantics

21 pages

RR-92-19

Ralf Legleitner, Ansgar Bernardi, Christoph

Klauck: PIM: Planning In Manufacturing using

Skeletal Plans and Features

17 pages

RR-92-20

John Nerbonne: Representing Grammar, Meaning and Knowledge

18 pages

RR-92-21

Jörg-Peter Mohren, Jürgen Müller

Representing Spatial Relations (Part II) -The

Geometrical Approach

25 pages

RR-92-22

Jörg Würtz: Unifying Cycles

24 pages

RR-92-23

Gert Smolka, Ralf Treinen:

Records for Logic Programming

38 pages

\section{RR-92-24}

Gabriele Schmidt: Knowledge Acquisition from Text in a Complex Domain

20 pages 
RR-92-25

Franz Schmalhofer, Ralf Bergmann, Otto Kühn, Gabriele Schmidt: Using integrated knowledge acquisition to prepare sophisticated expert plans for their re-use in novel situations

12 pages

\section{RR-92-26}

Franz Schmalhofer, Thomas Reinartz, Bidjan Tschaitschian: Intelligent documentation as a catalyst for developing cooperative knowledge-based systems

16 pages

RR-92-27

Franz Schmalhofer, Jörg Thoben: The model-based construction of a case-oriented expert system 18 pages

\section{RR-92-29}

Zhaohui Wu, Ansgar Bernardi, Christoph Klauck: Skeletel Plans Reuse: A Restricted Conceptual Graph Classification Approach

13 pages

\section{RR-92-30}

Rolf Backofen, Gert Smolka

A Complete and Recursive Feature Theory

32 pages

RR-92-31

Wolfgang Wahlster

Automatic Design of Multimodal Presentations 17 pages

RR-92-33

Franz Baader: Unification Theory

22 pages

\section{RR-92-34}

Philipp Hanschke: Terminological Reasoning and Partial Inductive Definitions

23 pages

RR-92-35

Manfred Meyer:

Using Hierarchical Constraint Satisfaction for

Lathe-Tool Selection in a CIM Environment

18 pages

RR-92-36

Franz Baader, Philipp Hanschke:

Extensions of Concept Languages for a

Mechanical Engineering Application

15 pages

RR-92-37

Philipp Hanschke: Specifying Role Interaction in Concept Languages

26 pages
RR-92-38

Philipp Hanschke, Manfred Meyer:

An Alternative to $\Theta$-Subsumption Based on

Terminological Reasoning

9 pages

RR-92-40

Philipp Hanschke, Knut Hinkelmann: Combining Terminological and Rule-based Reasoning for Abstraction Processes

17 pages

RR-92-41

Andreas Lux: A Multi-Agent Approach towards Group Scheduling

32 pages

\section{RR-92-42}

John Nerbonne:

A Feature-Based Syntax/Semantics Interface 19 pages

\section{RR-92-43}

Christoph Klauck, Jakob Mauss: A Heuristic driven Parser for Attributed Node Labeled Graph Grammars and its Application to Feature Recognition in CIM

17 pages

RR-92-44

Thomas Rist, Elisabeth André: Incorporating Graphics Design and Realization into the Multimodal Presentation System WIP 15 pages

\section{RR-92-45}

Elisabeth André, Thomas Rist: The Design of Illustrated Documents as a Planning Task 21 pages

\section{RR-92-46}

Elisabeth André, Wolfgang Finkler, Winfried Graf, Thomas Rist, Anne Schauder, Wolfgang Wahlster: WIP: The Automatic Synthesis of Multimodal Presentations

19 pages

\section{RR-92-47}

Frank Bomarius: A Multi-Agent Approach towards Modeling Urban Traffic Scenarios 24 pages

\section{RR-92-48}

Bernhard Nebel, Jana Koehler:

Plan Modifications versus Plan Generation:

A Complexity-Theoretic Perspective

15 pages

RR-92-49

Christoph Klauck, Ralf Legleitner, Ansgar Bernardi: Heuristic Classification for Automated CAPP 15 pages 
RR-92-50

Stephan Busemann:

Generierung natürlicher Sprache

61 Seiten

RR-92-51

Hans-Jürgen Bürckert, Werner Nutt:

On Abduction and Answer Generation through Constrained Resolution

20 pages

RR-92-52

Mathias Bauer, Susanne Biundo, Dietmar Dengler, Jana Koehler, Gabriele Paul: PHI - A Logic-Based Tool for Intelligent Help Systems 14 pages

RR-92-54

Harold Boley: A Direkt Semantic

Characterization of RELFUN

30 pages

RR-92-55

John Nerbonne, Joachim Laubsch, Abdel Kader Diagne, Stephan Oepen: Natural Language Semantics and Compiler Technology 17 pages

RR-92-58

Franz Baader, Bernhard Hollunder:

How to Prefer More Specific Defaults in

Terminological Default Logic

31 pages

RR-92-59

Karl Schlechta and David Makinson: On Principles and Problems of Defeasible Inheritance 14 pages

RR-93-02

Wolfgang Wahlster, Elisabeth André, Wolfgang

Finkler, Hans-Jürgen Profitlich, Thomas Rist:

Plan-based Integration of Natural Language and Graphics Generation

50 pages

RR-93-03

Franz Baader, Berhard Hollunder, Bernhard

Nebel, Hans-Jürgen Profitlich, Enrico Franconi:

An Empirical Analysis of Optimization Techniques for Terminological Representation Systems

28 pages

RR-93-05

Franz Baader, Klaus Schulz: Combination Techniques and Decision Problems for Disunification 29 pages

RR-92-60

Karl Schlechta: Defaults, Preorder Semantics and Circumscription

18 pages

\section{DFKI Technical Memos}

TM-91-12

Klaus Becker, Christoph Klauck, Johannes

Schwagereit: FEAT-PATR: Eine Erweiterung des

D-PATR zur Feature-Erkennung in CAD/CAM

33 Seiten

TM-91-13

Knut Hinkelmann: Forward Logic Evaluation:

Developing a Compiler from a Partially

Evaluated Meta Interpreter

16 pages

TM-91-14

Rainer Bleisinger, Rainer Hoch, Andreas Dengel:

ODA-based modeling for document analysis

14 pages

TM-91-15

Stefan Bussmann: Prototypical Concept Formation An Alternative Approach to Knowledge Representation 28 pages

\section{TM-92-01}

Lijuan Zhang: Entwurf und Implementierung eines Compilers zur Transformation von

Werkstückrepräsentationen

34 Seiten

TM-92-02

Achim Schupeta: Organizing Communication and Introspection in a Multi-Agent Blocksworld 32 pages

TM-92-03

Mona Singh:

A Cognitiv Analysis of Event Structure

21 pages

TM-92-04

Jürgen Müller, Jörg Müller, Markus Pischel.

Ralf Scheidhauer:

On the Representation of Temporal Knowledge

61 pages

TM-92-05

Franz Schmalhofer, Christoph Globig, Jörg Thoben:

The refitting of plans by a human expert

10 pages

TM-92-06

Otto Kühn, Franz Schmalhofer: Hierarchical skeletal plan refinement: Task- and inference structures

14 pages

TM-92-08

Anne Kilger: Realization of Tree Adjoining

Grammars with Unification

27 pages 


\section{DFKI Documents}

D-92-06

Hans Werner Höper: Systematik zur

Beschreibung von Werkstücken in der

Terminologie der Featuresprache

392 Seiten

\section{D-92-07}

Susanne Biundo, Franz Schmalhofer (Eds.): Proceedings of the DFKI Workshop on Planning 65 pages

\section{D-92-08}

Jochen Heinsohn, Bernhard Hollunder (Eds.):

DFKI Workshop on Taxonomic Reasoning

Proceedings

56 pages

\section{D-92-09}

Gernod P. Laufkötter: Implementierungsmöglichkeiten der integrativen Wissensakquisitionsmethode des ARC-TEC-Projektes 86 Seiten

D-92-10

Jakob Mauss: Ein heuristisch gesteuerter

Chart-Parser für attributierte Graph-Grammatiken 87 Seiten

\section{D-92-11}

Kerstin Becker: Möglichkeiten der Wissensmodellierung für technische Diagnose-Expertensysteme 92 Seiten

\section{D-92-12}

Otto Kühn, Franz Schmalhofer, Gabriele Schmidt: Integrated Knowledge Acquisition for Lathe Production Planning: a Picture Gallery (Integrierte Wissensakquisition zur Fertigungsplanung für Drehteile: eine Bildergalerie)

27 pages

D-92-13

Holger Peine: An Investigation of the Applicability of Terminological Reasoning to Application-Independent Software-Analysis 55 pages

D-92-14

Johannes Schwagereit: Integration von GraphGrammatiken und Taxonomien zur Repräsentation von Features in CIM 98 Seiten

\section{D-92-15}

DFKI Wissenschaftlich-Technischer Jahresbericht 1991 130 Seiten

\section{D-92-16}

Judith Engelkamp (Hrsg.): Verzeichnis von Softwarekomponenten für natürlichsprachliche Systeme 189 Seiten

\section{D-92-17}

Elisabeth André, Robin Cohen, Winfried Graf, Bob Kass, Cécile Paris, Wolfgang Wahlster (Eds.): UM92: Third International Workshop on User Modeling, Proceedings

254 pages

Note: This document is available only for a nominal charge of $25 \mathrm{DM}$ (or 15 US-\$).

D-92-18

Klaus Becker: Verfahren der automatisierten Diagnose technischer Systeme 109 Seiten

D-92-19

Stefan Dittrich, Rainer Hoch: Automatische, Deskriptor-basierte Unterstützung der Dokumentanalyse zur Fokussierung und Klassifizierung von Geschäftsbriefen 107 Seiten

\section{D-92-21}

Anne Schauder: Incremental Syntactic Generation of Natural Language with Tree Adjoining Grammars 57 pages

D-92-23

Michael Herfert: Parsen und Generieren der Prolog-artigen Syntax von RELFUN 51 Seiten

\section{D-92-24}

Jürgen Müller, Donald Steiner (Hrsg.):

Kooperierende Agenten

78 Seiten

D-92-25

Martin Buchheit: Klassische Kommunikationsund Koordinationsmodelle

31 Seiten

D-92-26

Enno Tolzmann.

Realisierung eines Werkzeugauswahlmoduls mit Hilfe des Constraint-Systems CONTAX 28 Seiten

D-92-27

Martin Harm, Knut Hinkelmann, Thomas Labisch: Integrating Top-down and Bottom-up Reasoning in COLAB

40 pages

D-92-28

Klaus-Peter Gores, Rainer Bleisinger: Ein Modell zur Repräsentation von Nachrichtentypen 56 Seiten 
302 now zindsisstoly

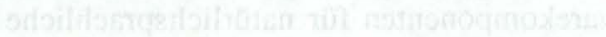

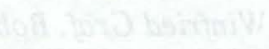

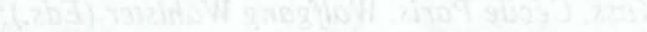

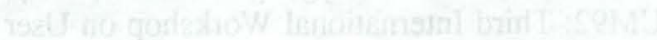

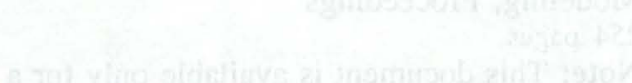

Pristera

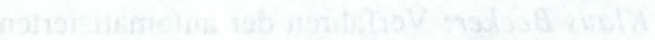

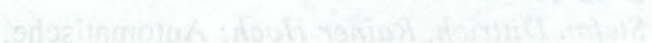

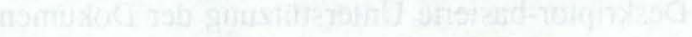

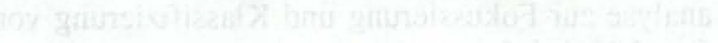

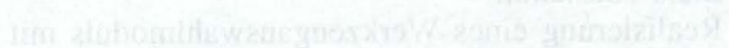

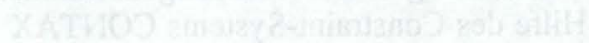

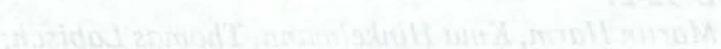

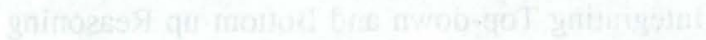
(41.) 1007) nit

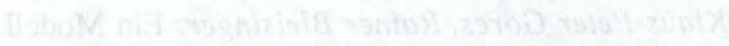

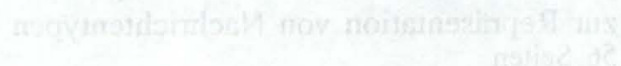





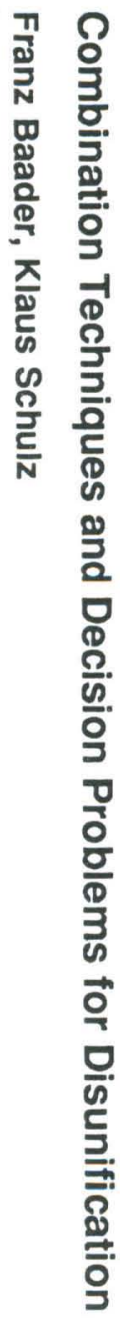

गౌo

(D)

(1)

อิ ग $\omega$

吾

익 